\title{
CONSTRUCGIÓN DE MODELOS VARIABLES EN DIALECTOLOGÍA: LA DISTRIBUCIÓN DE (S) EN LA GEOGRAFÍA FÓNICA DE MÉXICO
}

Es probable que buena parte de los problemas más interesantes planteados en la dialectología tradicional estén todavía por resolverse ${ }^{1}$. No hay que olvidar que el trabajo geolingüístico no nació, estrictamente hablando, para describir variedades espaciales, sino para discutir con datos de campo la plasmación de los procesos históricos de cambio lingüístico; nació, pues, como parte de la lingüística histórica. La desbordante complejidad de los datos provocó -y en parte sigue provocando- un cierto exceso descriptivo (podríamos llamarlo el exceso etnográfico) que desdibuja los objetivos lingüísticos primarios. La cuestión de las zonas dialectales de México es un buen ejemplo de problema tradicional que ha quedado a medio resolver, no sólo por falta de información, pues la que ya está disponible (el Atlas en primer término) se ha usado sólo en contados trabajos ${ }^{2}$, sino por la ausencia de engarces históricos $^{3}$ y por la casi nula aplicación de modelos lingüísticos que puedan poner orden en la abundancia de los datos dialectológicos, datos que suelen presentarse de forma en realidad mucho más ordenada y regular de lo que parece a primera vista. A esta vaguedad en el modelado variable puede llamársele carencia formal.

El propósito de este artículo es contribuir a evitar el exceso etnográfico y las carencias formales a la hora de trabajar con datos espaciales. Para ello, se analizarán los datos referentes a 56 varian-

1 Este trabajo forma parte de una investigación más amplia, en curso, destinada a redactar una Fonología variable del español de México. Agradezco los comentarios de Erik Willis (Indiana University) al manuscrito del artículo.

${ }^{2}$ Entre los trabajos dedicados al tema, destaca el de José G. Moreno DE Alba, La pronunciación del español en México, El Colegio de México, México, 1994.

3 Véase, ahora, Luis Fernando Lara, "Para la historia de la expansión del español por México”, NRFH, 56 (2008), 297-362. 
tes de la variable (s) intervocálica incluidos en un solo mapa del Atlas lingüístico de México, el número 26, y se propondrán algunas representaciones variables sobre los datos allí expuestos, en un intento de proponer un modelo de variación espacial relativamente abstracto o cuando menos dotado de cierta depuración factual ${ }^{4}$.

En ese sentido, se empieza por repasar los antecedentes, se describe la metodología empleada y se discute la organización y las características fónicas de las variantes registradas en el Atlas. A continuación, se analizan cuantitativamente los rasgos y las variantes involucradas, para establecer después varios modelos geolingüísticos y una representación fonético-fonológica sobre el conjunto de los datos.

\section{ANTECEDENTES}

A pesar de que (s) sea una de las variables fónicas más importantes para entender la organización dialectal y sociolingüística del español, se dispone de relativamente pocos estudios acerca de su constitución en el español mexicano. La mayoría de ellos, además, se refieren a los procesos de debilitamiento experimentados por (s) en posición de coda silábica ${ }^{5}$. Mi propósito aquí, sin embargo, es ocuparme de las características fónicas y de la

${ }^{4}$ Hace casi veinte años, en el contexto de sus comentarios al Atlas lingüístico de México, Manuel Alvar escribía lo siguiente, tomando como ejemplo, precisamente, el mismo mapa que se analiza en este artículo: "Si nos acodamos sobre el mapa 26, importantísimo por cuanto dice, vemos que las dificultades de lectura son inmensas. Y es que el número de informantes no hace sino enmarañar la transcripción: se nos habla de las exigencias del polimorfismo. Las sé, pero no creo que utilizar cuatro informantes en vez de uno nos dé todas las posibilidades del habla y en uno, con un largo cuestionario, se dan multitud de variantes" ("Ante el Atlas Lingüistico de México", NRFH, 39, 1991, p. 676). Este escrito intenta establecer entonces las líneas centrales de la lectura que no permite llevar a cabo la consulta directa del Atlas.

${ }_{5}$ Entre otros trabajos, conviene destacar las páginas que dedica MoreNO DE AlbA, en La pronunciación, al relajamiento de -s implosiva (pp. 74-104), el espacio concedido por Julio SERRANo al comportamiento de la variable entre inmigrantes sonorenses en la ciudad de México (Dialectos en contacto. Variación y cambio lingüistico en migrantes sonorenses, tesis de licenciatura, Escuela Nacional de Antropología e Historia, México, 2002, pp. 70-90), el trabajo de Rubí Ceballos ("Hacia un mayor consonantismo en la zona conurbada Veracruz-Boca del Río: el caso de la [s] implosiva”, en Líderes lingüísticos. Estudios de variación y cambio, ed. P. Martín, El Colegio de México, México, 2006, pp. 13-36) y el de Alejandra Espinosa Vázouez ("Variación del segmento /-s/ en El Ciruelo, Oaxaca”, en Fonología instrumental. Patrones fónicos 
distribución geográfica de la sibilante en posición de ataque en contexto intervocálico. Como se irá viendo a lo largo del trabajo, los procesos interesantes son de naturaleza diferente a los propios de las codas, y tienen que ver tanto con la raíz como con el modo, la actividad laríngea y la zona de articulación.

Moreno de Alba ha señalado que, aunque existen múltiples alófonos, la articulación más común de la (s) en el español de México es alveolar predorsal fricativa sorda ${ }^{6}$, variante muy frecuente también en otras áreas del español americano, caracterizada por emplear el predorso de la lengua como articulador activo, apoyado sobre los alveolos superiores, que funcionan como articulador pasivo ${ }^{7}$. La diferencia con la (s) castellana consiste, en el terreno articulatorio, en que en esta variedad de habla es el ápice de la lengua el que actúa como articulador activo. Y según el órgano activo sea el predorso o el ápice, la posición de la lengua varía, de forma que es convexa o cóncava, de modo respectivo ${ }^{8}$.

y variación, eds. E. Herrera Z. y P. Martín, El Colegio de México, México, 2008, pp. 107-128).

6 En la p. 21 del Atlas, al pie del "Alfabeto fonético", se anota en referencia a la dorsoalveolar convexa fricativa sorda que "en multitud de casos, la llamada /s/ «mexicana»es, en cuanto a su zona pasiva de fricción, alveolodental; sería, pues, dorsoalveolo-predorsodental (o dorso-dentoalveolar) fricativa sorda" (Atlas lingüistico de México, dir. J.M. Lope Blanch, El Colegio de México-UNAM-F.C.E., México, 1990-2000).

7 En 1921, HenríQuez Ureña señalaba diferentes matices de las $s$ americanas: "otro, muy distinto, es la ese de la ciudad de México, sonido prolongado en que la punta de la lengua se apoya más o menos en los incisivos inferiores" (“Observaciones sobre el español de América”, RFE, 8, 1921, 357-390, cito por la ed. de 1993: La división dialectal del español de América, ed. F. Moreno Fernández, Universidad de Alcalá, Alcalá de Henares, 1993, p. 51). "Son menos sibilantes, y se acercan más al timbre castellano, como de sh, la ese de Chihuahua... muy distinta de la que se oye en la capital de la República” (id., n. 43).

${ }^{8}$ Moreno de Alba (op. cit., p. 77). Es conocido el pasaje de Navarro TomÁs: "hay entre la s española y la $s$ corriente en otros idiomas una diferencia importante; esta diferencia se manifiesta, principalmente, en la posición de la punta de la lengua, la cual, en la s española, se eleva... estrechándose, por su línea más exterior y más próxima al ápice, contra los alvéolos superiores, mientras que en la $s$ común francesa, italiana, alemana, etc., la parte de la lengua que forma la articulación es esencialmente el predorso, quedando pasivo el ápice, el cual desciende a veces y se apoya más o menos contra los incisivos inferiores. En una y otra articulación, la estrechez, de la cual resulta la fricación, viene a formarse sobre el mismo punto de la boca, oscilando, según los casos, entre los alvéolos y los dientes superiores; pero la parte de la lengua que forma esa estrechez, en la s española es más exterior y apical que en las demás variantes indicadas. Junto con esto, en la sespañola, la posición del predorso, detrás del punto de articulación, es... ligeramente cóncava, mientras que en 
Es obvio que para establecer en más detalle ciertas características articulatorias y acústicas, será necesario hacer algunos estudios acústicos y electropalatogáficos de cuando menos las principales variedades documentadas en México. Las variantes laminales, frente a las apicales, se caracterizan por una menor intensidad global, por tener el primer pico prominente en el espectro más elevado y por tener también más alto el comienzo de la mayor intensidad de ruido; la apical es más sibilante (o estridente) que la laminal ${ }^{9}$.

Las Figuras 1 y 2 ilustran estas diferencias; se trata de dos pronunciaciones de /s/ en la palabra salsa, la primera mexicana y la segunda castellana:
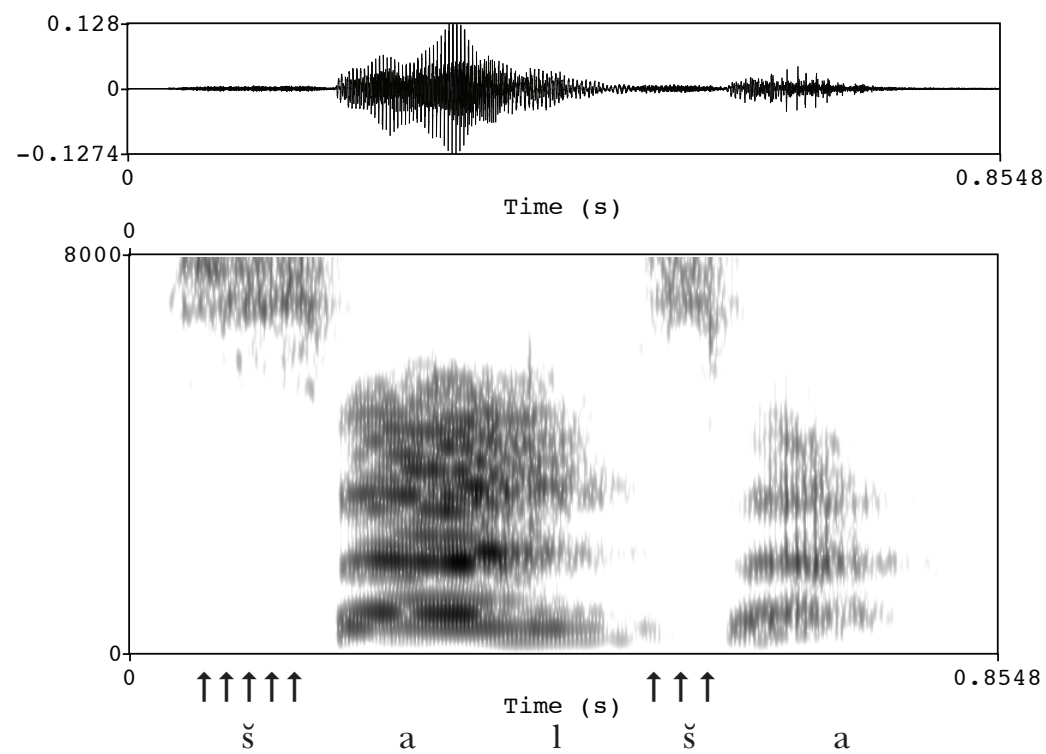

Figura 1. Oscilograma y espectrograma de la palabra salsa en voz femenina de la ciudad de México.

el tipo general, francés, italiano, etc., es más o menos convexa. De aquí resulta que el timbre de estos sonidos es bastante distinto: el de la $s$ española es más grave y palatal que el de la otra $s . .$. La $s$ andaluza y la de una gran parte de la América española es de tipo predorsal, aunque con notables variantes entre unos países y otros" (Manual de pronunciación española, 21 $1^{\mathrm{a}}$ ed., CSIC, Madrid, 1982, pp. 106-107). Se comenta también que "en algunas variantes de $s$ predorsal, plana o convexa, la punta de la lengua suele intervenir también en la formación de la estrechez dentoalveolar, pero sin que este hecho figure como una circunstancia especial en la articulación" (ibid., p. 107, n. 1).

9 Cf. Martínez Celdrán y Fernández Planas (Manual de fonética española. Articulaciones y sonidos del español, Ariel, Barcelona, 2007, pp. 110-111). En el 
Las /s/ de la Figura 1 se han transcrito como dorso-alveola-

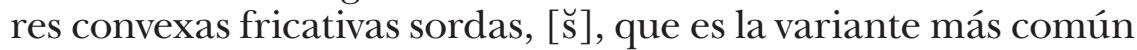
en el Atlas lingüistico -más que la lámino-alveolar plana fricativa sorda $[\bar{s}]$. El ruido de la turbulencia fricativa comienza alrededor de los $6500 \mathrm{~Hz}$ en los dos casos, altura que contrasta claramente con el ejemplo expuesto en la Figura 2:
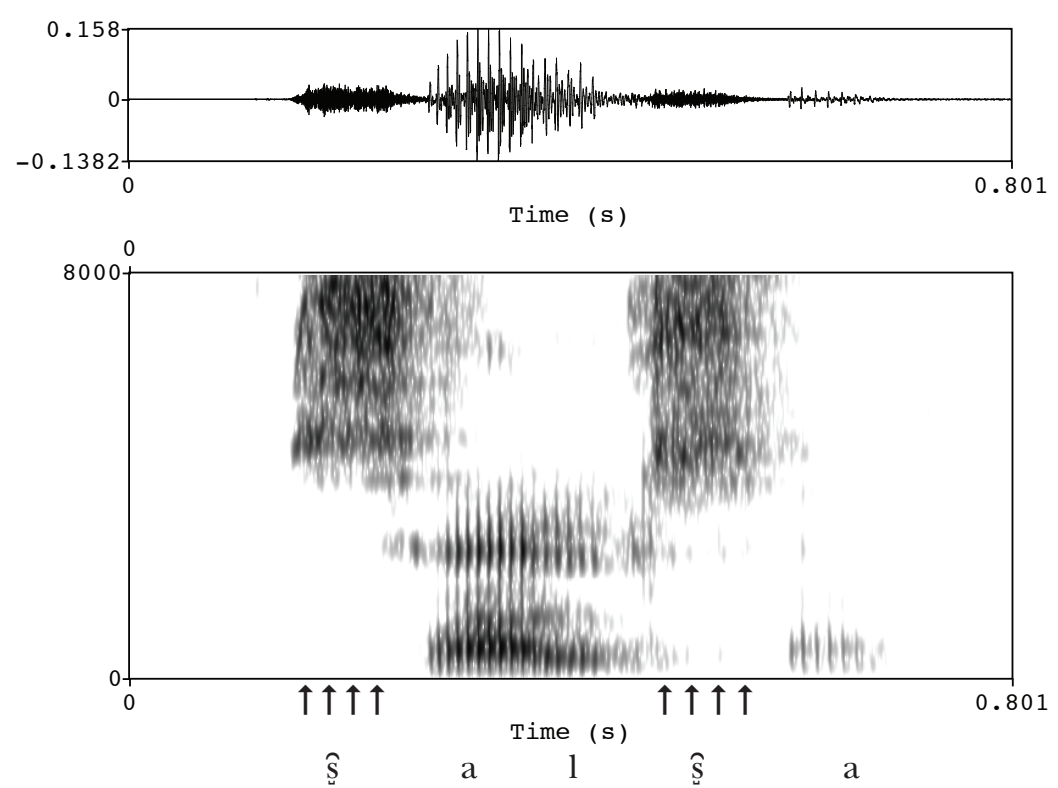

Figura 2. Oscilograma y espectrograma de la palabra salsa en voz masculina de Madrid

Como puede verse, la Figura muestra dos ejemplos con [ $[\hat{\mathrm{S}}]$ ápico-alveolar cóncava fricativa sorda; el ruido comienza ahora mucho más bajo que en el ejemplo anterior, alrededor de los $3500 \mathrm{~Hz}$ en las dos ocurrencias.

Los datos generales de /s/ intervocálica en el Atlas fueron examinados -junto con los de $/ \overline{\mathrm{t}} / \mathrm{y} / \mathrm{x} /$, de forma que se considera las relaciones entre ellos- por López Chávez en un trabajo publicado en $1986^{10}$, tras el análisis de más de 700 cintas

ejemplo que ofrecen en la Figura 2.70, el alófono apical presenta -28 dB de intensidad global, por $-39 \mathrm{~dB}$ para la laminal (argentina), el primer pico prominente ostenta $4622 \mathrm{~Hz}$ y $-45 \mathrm{~dB}$ en la apical, por $6230 \mathrm{~Hz}$ y $-56 \mathrm{~dB}$ en la laminal, y el comienzo de la mayor intensidad de ruido está en $3500 \mathrm{~Hz}$ para la apical y en 5000 para la laminal.

10 “/s/, / c / y /x/ intervocálicas en la República mexicana", en Actas del II Congreso Internacional sobre el español de América (Ciudad de México, 
magnetofónicas grabadas en 193 localidades. Las transcripciones estrechas se agrupan allí en cuatro variantes: una predorsodentoalveolar convexa [s], que aparece en $64.50 \%$ de los casos; otra que agrupa los alófonos con predominio de fricación dental, [s] ], que suma $14.30 \%$; una tercera para las variantes con predominio de fricación apicoalveolar y resonancia palatal, [is], que llega a $18.90 \%$; y una última para las variantes aspiradas, [h], apenas con $2.20 \%^{11}$.

\section{Precisiones metodológicas}

Es importante aclarar que este trabajo parte de dos supuestos metodológicos: 1) en general, no se cuestionan las transcripciones incluidas en el Atlas, sino que se acepta el registro allí expuesto; 2) se estandariza el alfabeto fonético, acercando la transcripción a formas más afines al Alfabeto Fonético Internacional y, lo que es más importante, se intenta normalizar la descripción articulatoria de cada variante mencionada, buscando hacer más coherentes las observaciones que se deducen de cada variante particular y del cuadro que se desprende de su conjunto. Ambos supuestos implican correr varios riesgos analíticos que en este momento me parecen inevitables ${ }^{12}$.

27-31 de enero de 1986), ed. J.G. Moreno de Alba, UNAM, México, 1986, pp. 327-329.

${ }^{11}$ En apariencia, no se publicaron junto con el trabajo algunos mapas adjuntos mencionados en texto, lo cual dificulta la interpretación del escrito; tampoco los signos fonéticos son muy nítidos. Se mencionan algunos hechos de interés, como que la aspiración de /s/ intervocálica se da en el noroeste de México; parece también que las variantes con resonancia palatal son más comunes en el sur. Tampoco es fácil la comparación de los porcentajes globales. En el Cuadro 10 (infra) he desglosado los datos según el lugar articulatorio. Las formas dorso-alveolares suman allí $64.40 \%$, lo que coincide bastante bien con $64.50 \%$ de predorsoalveolares de López CHÁvez; si se suman las interdentales, ápico-dentales y predorso-dentales, el Cuadro 10 se salda con $14.68 \%$, de nuevo cercano a $14.30 \%$ de las llamadas dentales genéricamente en su art. cit.; la suma de postalveolares y faríngeas en el Cuadro es $2.44 \%$, de nuevo no lejos de $2.20 \%$ de las aspiradas en la cuenta de López Chávez. Por fin, las lámino-alveolares del Cuadro suponen 18\%, no lejano en el número a $18.90 \%$ de las formas con predominio fricativo apicoalveolar y resonancia palatal del trabajo ahora citado.

12 De forma ideal, un mejor análisis debería descansar en la consideración directa de los materiales, en forma acústica o electropalatográfica, para establecer las finas distinciones propias de las más de cincuenta variantes 
Más allá de estas dos precisiones, se ha procedido de la siguiente manera. Como es bien sabido, el Atlas lingüístico de México contiene una serie de mapas sintéticos que condensan una enorme cantidad de información, fruto de la anotación de 754 grabaciones correspondientes a informantes procedentes de 193 localidades diferentes, de forma que en cada punto se estima la frecuencia de las variantes que se presentan en él ${ }^{13}$. De manera paradójica, se ha observado en varias ocasiones que esa misma gran cantidad de información dificulta la lectura de las cartas geolingüísticas ${ }^{14}$; como sea, es probable que hubiera sido más productivo presentar los materiales en forma de una base de datos.

El Mapa 26 (preparado por G. Cantero, G. Gardner y J. López) es uno de los más ricos en cuanto a información se refiere, pues incluye 1329 registros diferentes -es decir, un promedio de casi siete formas distintas por localidad-, de las que se estima su dispersión, tanto global en cada punto como por cada uno de los informantes entrevistados. A los efectos de este trabajo, tras adaptar las transcripciones del Atlas a las formas del AFI, como se ha dicho ${ }^{15}$, se trasladaron todos los materiales incluidos en el Mapa a una base de datos, que es el punto de partida para el análisis cuantitativo ${ }^{16}$ y cualitativo llevado a cabo.

mencionadas en el Mapa 26. Lo primero es complejo -hoy por hoy las grabaciones del Atlas ni siquiera están digitalizadas en su conjunto, y las pocas que he podido examinar en formato digital dejan cuando menos en suspenso la posibilidad de un análisis acústico muy preciso, más allá de algunos grandes rasgos-; lo segundo, por razones obvias, es imposible. Queda pendiente, entonces, el levantamiento de nuevos materiales sonoros a lo largo y ancho del país, con nuevos métodos que permitieran la confección de un Corpus oral del español de México (COEM). Por lo demás, el trabajo de los redactores del Mapa ahora considerado es extraordinario y merece por lo pronto el mayor respeto.

${ }^{13}$ Cf. Lope Blanch, "Dialectología mexicana y sociolingüística”, NRFH, 23 (1974), 1-34 y el propio Atlas.

14 Cf. M. Alvar, art. cit., p. 676.

15 Cf. P. Martín Butragueño ("Alfabeto fonético", ms.) para la adaptación completa. En el caso de (s), ha sido necesario introducir, para respetar la precisión de las anotaciones del Atlas, diacríticos que refieren a la posición convexa, plana o cóncava de la lengua; también se han incluido combinaciones de diacríticos para expresar todos los detalles fonéticos. Véase el Apéndice al final del artículo para la lista completa de signos fonéticos; se incluyen los signos del Atlas, la adaptación propuesta y una descripción articulatoria normalizada.

${ }^{16}$ La cuantificación se llevó a cabo por medio de SPSS (SPSS. Statistical Package for the Social Sciences, versión 13 para Windows, SPSS Inc.) y de Goldvarb X (David Sankoff, Sali A. Tagliamonte y E. Smith, Goldvarb X. 
Un punto central en la metodología es la decisión de apoyarse en un modelo geográfico constituido por cinco secciones, semejante al empleado en dos trabajos previos -sobre (j) y sobre $\left.\widehat{\left(\mathrm{t} \int\right.}\right)^{17}$-, en los que tal división había demostrado ser bastante productiva. Por supuesto, las cinco zonas encierran una simplificación geográfica y su valor de entrada es funcionar sólo como hipótesis, previa a la delimitación futura de regiones más precisas. Las regiones quedan entonces ordenadas así: sureste (Campeche, Chiapas, Quintana Roo, Tabasco, Yucatán) ${ }^{18}$; centro-este $^{2}$ (Distrito Federal, Oaxaca, Puebla, Tlaxcala, Veracruz); centrooeste (Aguascalientes, Colima, Guanajuato, Guerrero, Hidalgo, Jalisco, México, Michoacán, Morelos, Querétaro); noreste (Coahuila, Nuevo León, San Luis Potosí, Tamaulipas); noroeste (Baja California, Baja California Sur, Chihuahua, Durango, Nayarit, Sinaloa, Sonora, Zacatecas ${ }^{19}$.

\section{CaracterizaCión DE LAS VARIANTES DE (s)}

El Cuadro 1 expone una visión somera de las 56 variantes de (s) documentadas en el Mapa 26, diferentes por lo pronto según varias dimensiones: zona de articulación, región lingual involucrada, disposición de la lengua, modo articulatorio, lateralización, nasalización y mayor o menor definición de la realización (hipoarticulación).

Como puede apreciarse, las 1329 observaciones sobre (s) corresponden a catorce lugares articulatorios diferentes ${ }^{20}$, orde-

A multivariate analysis application, University of Toronto-University of Ottawa, 2005; puede obtenerse en http://individual.utoronto.ca/tagliamonte/Goldvarb/GV_index.htm).

17 P. Martín Butragueño, "Estructura del yeísmo en la geografía fónica de México", ms., y "Regularidad y excepcionalidad del cambio lingüístico: el caso de (t⿱⺈) en la geografía fónica de México", ms., respectivamente.

18 Lope Blanch ("Una nota sobre la sibilante africada", ALM, 27, 1989, 263-268. También en Ensayos sobre el español de América, UNAM, México, 1993, pp. 191-197) ya se refería a la gran pluralidad de soluciones en el español yucateco, desde variantes ápico-alveolares cóncavas -y menciona a este propósito como ejemplo a García Fajardo (Fonética del español de Valladolid, Yucatán, UNAM, México, 1984, pp. 58 ss.)- a posdentales ciceantes (pp. 191192 del trabajo de Lope).

19 Ya se ha mencionado que López Chávez (art. cit.) señala rasgos peculiares del noroeste, como la aspiración.

${ }^{20}$ Además, hubo algunos casos de elisión. 


\section{Cuadro 1}

Variantes de (s) presentes en el Mapa 26 del Atlas

\begin{tabular}{|c|c|c|c|}
\hline LUGAR & $\begin{array}{l}\text { región } \\
\text { lingual }\end{array}$ & $\begin{array}{l}\text { disposición de } \\
\text { la lengua }\end{array}$ & variantes \\
\hline interdental ( 5 casos) & & & {$[\theta](3),[\underset{\mp}{\theta}](1),\left[\theta_{+}\right](1)$} \\
\hline $\begin{array}{l}\text { postinterdento-dental } \\
(92)\end{array}$ & & & {$[\theta](81),[\ddot{\theta}](9),[\widehat{\mathrm{t}} \theta](1),[\hat{\theta} \theta](1)$} \\
\hline dento-interdental (17) & & & {$\left[s_{n}^{\theta}\right](17)$} \\
\hline \multirow[t]{3}{*}{ dental (234) } & \multirow{2}{*}{$\begin{array}{l}\text { ápice } \\
\text { (109) }\end{array}$} & plana (106) & 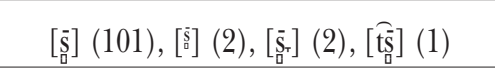 \\
\hline & & $\begin{array}{l}\text { levemente } \\
\text { cóncava }(3)\end{array}$ & {$\left[\begin{array}{l}(s) \\
\text { s.j }\end{array}\right](3)$} \\
\hline & $\begin{array}{l}\text { predorso } \\
(125)\end{array}$ & $\begin{array}{l}\text { convexa } \\
(123)\end{array}$ & 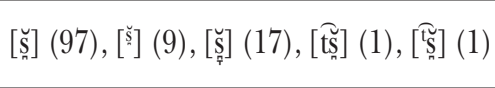 \\
\hline dento-alveolar (10) & & & {$\left[\mathrm{I}_{\mathrm{s}}\right](10)$} \\
\hline $\begin{array}{l}\text { dento-alveolar } \\
\text { palatalizada (4) }\end{array}$ & & & {$\left[\overline{\mathrm{ts}}^{\mathrm{j}}\right](1),\left[{ }^{\mathrm{T}_{\mathrm{s}}}\right](3)$} \\
\hline \multirow[t]{6}{*}{ alveolar (851) } & \multirow[t]{2}{*}{ ápice (9) } & cóncava (6) & {$[\hat{\mathrm{s}}](6)$} \\
\hline & & $\begin{array}{l}\text { levemente } \\
\text { cóncava (3) }\end{array}$ & {$\left[\left(\hat{s}^{\prime}\right](3)\right.$} \\
\hline & \multirow{3}{*}{$\begin{array}{l}\text { lámina } \\
(304)\end{array}$} & plana (184) & 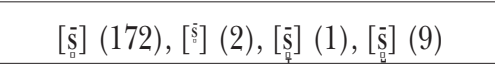 \\
\hline & & $\begin{array}{l}\text { plano- } \\
\text { cóncava (39) }\end{array}$ & 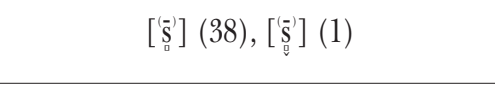 \\
\hline & & $\begin{array}{l}\text { levemente } \\
\text { cóncava }(81)\end{array}$ & {$\left[\begin{array}{c}\left(\begin{array}{c}s \\
\mathrm{~s}\end{array}\right] \\
\mathrm{a}\end{array}\right](81)$} \\
\hline & $\begin{array}{l}\text { dorso } \\
(538)\end{array}$ & $\begin{array}{l}\text { convexa } \\
(538)\end{array}$ & 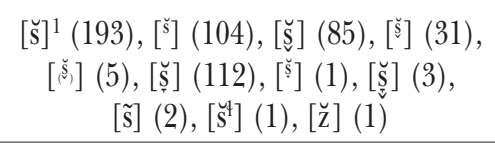 \\
\hline alveolar palatalizada (9) & dorso (9) & convexa (9) & {$[\breve{s}](9)$} \\
\hline postalveolo-alveolar (4) & & & {$\left[\int^{s}\right](4)$} \\
\hline postalveolar (36) & & & {$\left[\int\right](32),\left[\lfloor](1),[3](2),\left[\int\right]\right](1)$} \\
\hline velo-faríngea (1) & & & {$\left[\mathrm{x}^{\mathrm{\hbar}}\right](1)$} \\
\hline faríngeo-alveolar (9) & & & {$\left[\hbar^{s}\right](7),\left[{ }^{\hbar_{s}}\right](2)$} \\
\hline faríngeo-postalveolar (3) & & & {$[\hbar !](3)$} \\
\hline faríngea (49) & & & {$[\hbar](22),\left[^{\hbar}\right](6),[\uparrow](13),\left[^{\natural}\right](8)$} \\
\hline elisiones (5) & & & {$[\varnothing](5)$} \\
\hline
\end{tabular}

${ }^{1}$ Se documentó un caso con oclusión glotal previa: [?̌̌] $]$. 
nados a lo largo de un eje antero-posterior que va desde la zona interdental a la faríngea. En las áreas más prolíficas -siguiendo en esto la descripción fonética articulatoria del Atlas-, es decir, en las zonas dental y alveolar, el Cuadro 1 indica la región lingual participante en la articulación y la disposición de la lengua. De esa forma, el ápice de la lengua puede ser articulador activo cuando ésta se manifiesta plana, levemente cóncava o cóncava; la lámina puede actuar al tiempo que el cuerpo lingual es plano, plano-cóncavo o levemente cóncavo; por fin, la acción del predorso o del dorso está asociada a una disposición convexa. Se produce, pues, lo esperado en general: la actuación articulatoria de las regiones más anteriores de la lengua están asociadas a una conformación plana o (levemente) cóncava, mientras que la utilización de las más posteriores pide una postura convexa. La cuarta columna del Cuadro, por su parte, desglosa el conjunto de formas variantes anotadas, sin discriminar todavía los casos dotados de cierta sonoridad, las realizaciones no fricativas y los alófonos menos definidos en su articulación ${ }^{21}$.

El Cuadro 1 incluye también el recuento de variantes en los 193 puntos del Atlas lingüistico de México; se ha anotado, entre paréntesis, el número de ocasiones en que aparece cada variante, así como las sumas de casos según estos se van agrupando hacia la izquierda, hasta llegar a las cantidades parciales propias de cada zona articulatoria. En esos términos, las cuatro familias de variantes más comunes (las que superan las 100 documentaciones teniendo en cuenta el lugar articulatorio y la región lingual actuante) son las que se anotan en el Cuadro 2:

\section{CuAdro 2}

Familias de variantes de (s) más difundidas

\begin{tabular}{c|cc}
\hline LUGAR & $F$ & $f(F / N=1329)$ \\
\hline dorso-alveolares & 538 & 0.404 \\
lámino-alveolares & 304 & 0.228 \\
predorso-dentales & 125 & 0.094 \\
ápico-dentales & 109 & 0.082 \\
totales & 1076 & 0.809 \\
\hline
\end{tabular}

${ }^{21}$ El papel de estas distinciones es variable según las lenguas. La precisión entre formas apicales y laminales puede quedar en suspenso en determinadas ocasiones. Es lo que ocurre -a cierto nivel de abstracciónen lenguas como el inglés, donde la [s] alveolar puede ser apical o lami- 
Las formas más difundidas por la geografía fónica de México son, entonces, las [s] alveolares, que juntas suman dos terceras partes de las documentaciones $(\mathrm{f}=0.633)$, más comunes las dorsales que las laminales. Siguen a éstas las dentales, del tipo [s] que andan cerca de aparecer una de cada cinco veces $(\mathrm{f}=0.176)$, sean predorsales o apicales. Estas cantidades son, por otra parte, algo relativas, pues el cálculo más preciso ha de considerar el papel de la frecuencia ponderada en cada punto, y no sólo el número total de menciones (infra).

Para poner un poco más de orden en el conjunto de las variantes es necesario introducir una representación fonológica un poco más detallada; en este trabajo se empleará la establecida por Hall para los rasgos fónicos ${ }^{22}$ :

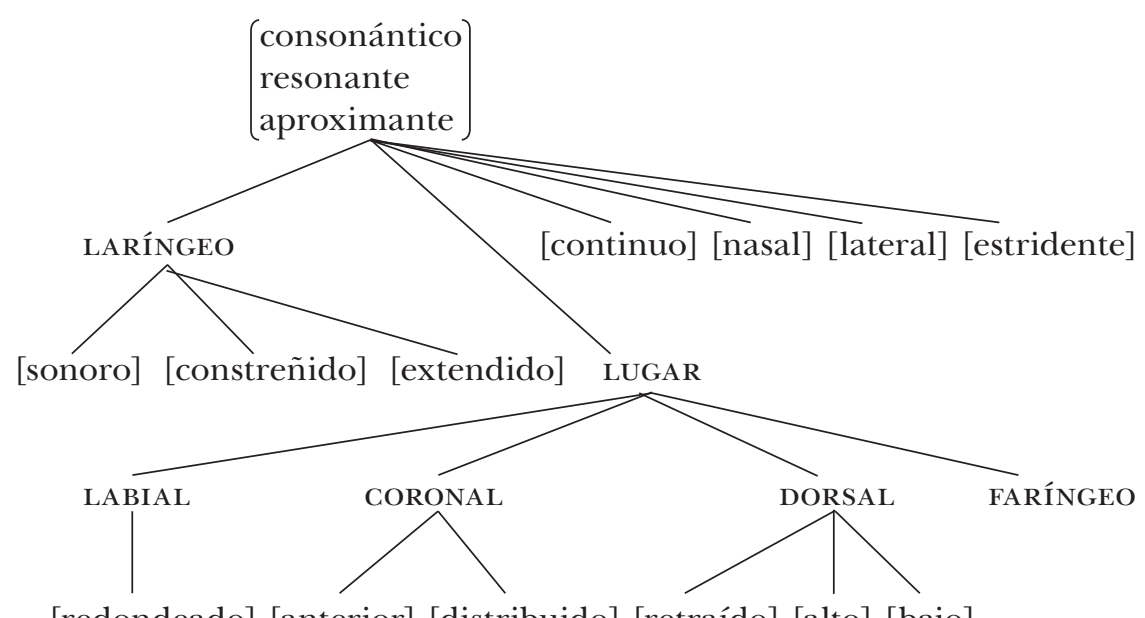

[redondeado] [anterior] [distribuido] [retraído] [alto] [bajo]

FIGURA 3. Representación jerárquica de los rasgos fónicos.

Como puede apreciarse, en esta propuesta los rasgos radicales son [consonántico], [resonante] y [aproximante]; de la raíz surgen luego seis líneas, dos de ellas correspondientes a los nodos LARÍNGEO y LUGAR, y cuatro más propias de seis rasgos

nal; en otras, como en toda (una lengua dravídica meridional), en cambio, la alveolar sibilante es siempre laminal, [s] (cf. PETER LADEFoged \& IAN MadDieson, The sounds of the world's languages, Blackwell, Oxford, 1996, p. 164).

${ }^{22}$ Fuente: T.A. HALl, "Segmental features", en The Cambridge handbook of phonology, ed. P. de Lacy, Cambridge University Press, Cambridge, 2007, p. 313. 
que cuelgan directamente de la raíz de la representación: [continuo], [nasal], [lateral] y [estridente]. El nodo LARÍNGEO, por su parte, vincula los rasgos [sonoro], [constreñido] y [extendido], y el nodo LUGAR ordena cuatro subnodos que anclan las diferentes zonas articulatorias: LABIAL, dotado además de un posible rasgo de [redondeado]; CORONAL, descrito en más detalle por medio de [coronal] y [distribuido]; DORSAL, especificado con [retraído], [alto] y [bajo]; y FARÍngEO. Respecto a estos rasgos, y en particular en lo que toca a los que ahora es pertinente considerar, las formas variantes del Cuadro 1 quedan caracterizadas de la siguiente forma (dejando aparte las elisiones):

\section{CUADRO 3}

Caracterización de las variantes según los rasgos ubicados en la raíz

\begin{tabular}{|c|c|c|}
\hline & {$[+]$} & {$[-]$} \\
\hline [consonántico] & Todas las variantes. & \\
\hline [resonante] & & Todas. \\
\hline [aproximante] & 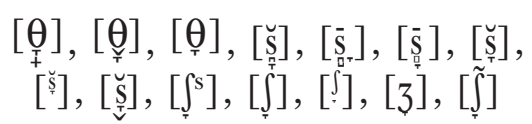 & Las demás. \\
\hline
\end{tabular}

Todas las variantes se caracterizan por poseer los rasgos [+consonántico] y [-resonante]; es decir, no se documentan casos de vocalización ni de laringización ${ }^{23}$; ni tampoco de lateralización plena (aunque sí hay algún caso de lateralización parcial), nasalización plena (sí parcial) ni rotacismo (en ningún caso, frente a lo que llega a ocurrir en otras variedades de habla $)^{24}$. Entre todas las documentaciones incluidas en el

${ }^{23}$ La laringización se produciría con [h] y con [2], que son ambas [-consonantes], [-resonantes], y [+extendido] y [-constreñido] la primera, y [-extendido] y [+constreñido] la segunda en el nodo LARÍnGEO, carentes sin embargo de LUGAR de articulación. Las transcripciones del Atlas, no obstante, apuntan a la presencia de aspiraciones faríngeas, no de aspiraciones laríngeas. Hubo, con todo, un único caso, ya mencionado en una nota previa, transcrito como [?̌s] , es decir, con la laríngea oclusiva sorda.

${ }^{24}$ Para $\mathrm{s} \rightarrow \mathrm{r}$, véase el trabajo de Francisco Moreno Fernández, "El paso -s > -؟ en español", Journal of Hispanic Research, 1 (1992-93), 17-34. Los procesos de nasalización, lateralización y rotacismo implican la activación de [+resonante]. Dado su carácter completamente marginal en la muestra (como en el caso mencionado en la nota anterior), y dada la permanencia de rasgos básicos de (s), no ha parecido necesario marcarlo mayormente respecto a este rasgo. 
Mapa 26, se ha considerado aquí que en 187 ocasiones ( $f=0.140)$ podría tratarse de formas aproximantes; entre ellas la más abundante, con mucho, sería la [șّ], una forma dorso-alveolar convexa aproximante sorda, que llega a aparecer en 112 menciones ${ }^{25}$. La atribución del rasgo [+aproximante] a estas variantes es discutible, y es necesario detenerse un momento en ello.

Las transcripciones del Atlas que emplean el signo ['] se asocian a dos tipos de glosa, "ligeramente abierta" -como en $\left[\mathrm{s}^{\mathrm{s}}\right]-\mathrm{y}$ "relajada, poco tensa" -tal es el caso de $\left[\theta^{*}\right]-$; se trata de varian-

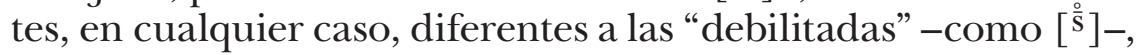
expresadas estas últimas por medio de voladitas. Se propone etiquetar las variantes ahora discutidas como aproximantes bajo el razonamiento de que una fricativa abierta es una aproximante; se trataría, por tanto, de formas intermedias entre las fricativas dentales, alveolares y postalveolares, y la aproximante [.]. Los sonidos fricativos, como es bien sabido, son aquellos que producen una corriente de aire turbulento en el canal vocal. El gesto articulatorio propio de las fricativas -y especialmente el de las sibilantes-requiere, por otra parte, de una gran precisión, como señalan Ladefoged y Maddieson ${ }^{26}$; la pérdida de esta precisión conduce a soluciones aproximantes.

\section{Cuadro 4}

Rasgos que cuelgan de la raíz

\begin{tabular}{|c|c|c|}
\hline & {$[+]$} & {$[-]$} \\
\hline$[$ continuo $]$ & Las demás. & 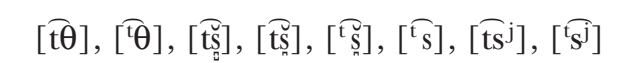 \\
\hline [nasal] & {$[\tilde{\mathrm{s}}],\left[\tilde{\int}\right]$} & Las demás. \\
\hline [lateral] & {$\left[\breve{s}^{\ddagger}\right]$} & Las demás. \\
\hline [estridente] & Las demás. & 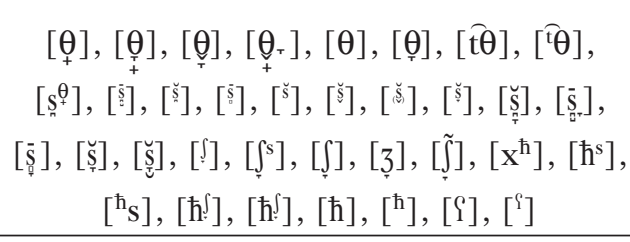 \\
\hline
\end{tabular}

Las formas marcadas como [-continuas] son las soluciones africadas. Aunque no son muchas (suman un total de diecinue-

25 Para las cantidades propias de cada una de las variantes, en este y en los siguientes Cuadros, véase el Cuadro 1, supra.

${ }_{26}$ Op. cit., p. 137. 
ve documentaciones), no dejan de ser llamativas; refuerzan la idea tradicional de la tensión de la (s) mexicana ${ }^{27}$. En la mayoría de los casos se trata de alófonos con el momento oclusivo breve: sólo en cuatro ocasiones los transcriptores del Atlas han anotado una oclusión plena. La forma más común entre las variantes africadas, de hecho, es una dento-alveolar sorda con el momento oclusivo breve [ $\left.{ }^{\mathrm{t}} \mathrm{s}\right]$. El papel de [nasal] y [lateral] es escaso, pero se manifiesta en la presencia de tres formas nasalizadas y una lateralizada.

Se ha contabilizado 506 casos de formas no estridentes. Entre las [-estridentes], destaca la postinterdento-dental fricativa sorda $[\theta]$, entre otras variantes interdentalizadas; también carecen de estridencia las diferentes aspiraciones faríngeas documentadas. A estas formas anteriores y posteriores se han sumado las articulaciones centrales poco definidas, por considerar que su debilidad difícilmente permitiría sostener el carácter [+estridente] ${ }^{28}$. También se ha considerado [-estridentes] todos los casos de [+aproximantes], pues la salida del aire sin fricción ${ }^{29}$ imposibilita por ello mismo la presencia de una fricción estridente ${ }^{30}$; entre estas últimas la más común, como ya se

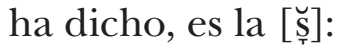

CUADro 5

Rasgos del nodo LARÍNGEO

\begin{tabular}{|c|c|c|}
\hline & {$[+]$} & {$[-]$} \\
\hline [sonoro] & 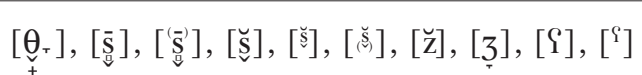 & Las demás. \\
\hline [constreñido] & & Todas. \\
\hline [extendido] & & Todas. \\
\hline
\end{tabular}

27 Cf. Raúl Ávila ("Realizaciones tensas de /s/ en la ciudad de México", ALM, 11, 1975, 235-239) y Lope BlANCH ("Una nota sobre la sibilante..."). Debe considerarse que el contexto intervocálico no es especialmente favorecedor de las soluciones [-continuas]. Ávila había señalado, para la Ciudad

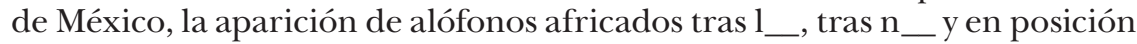
inicial absoluta, \#_. Lope Blanch amplía las observaciones refiriéndose

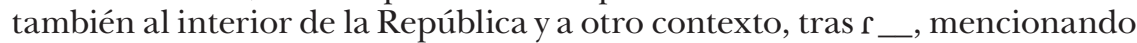
tanto materiales recogidos en la capital como en el Atlas. La africación se registra en todos los puntos de la carta, en los contextos mencionados, en el orden $n_{-}>1_{\ldots}>\# \_>r_{\ldots}$.

28 Agradezco la sugerencia a Esther Herrera.

29 Cf. T.A. Hall, art. cit., p. 316.

30 Véase también la clasificación de los modos de articulación en P. Ladefoged e I. Maddieson (op. cit., p. 371, Tabla 11.2). 
El nodo LARÍNGEO desempeña un papel modesto en la organización de los datos. Hubo 159 documentaciones de casos sonoros, sonorizados o levemente sonorizados. De hecho, las formas propiamente sonoras suponen una fracción relativamente reducida del total del material; sólo la faríngea fricativa sonora [§] es abundante, sea en su forma plena, sea en su forma poco definida o debilitada. Más comunes son las formas sonorizadas, en especial la dorso-alveolar convexa fricativa sonorizada [ $\breve{s}$ ], tanto plena como poco definida ${ }^{31}$ :

\begin{tabular}{|c|c|}
\hline & $\begin{array}{c}\text { CUADRo } 6 \\
\text { Rasgos del nodo LUGAR }\end{array}$ \\
\hline & $\checkmark$ \\
\hline LABIAL & Ninguna. \\
\hline CORONAL & {$[\underset{+}{\theta}],[\underset{\mp}{\theta}],[\underbrace{}_{+}],[\theta],[\hat{\theta}],[\widehat{\mathrm{t}} \theta],\left[{ }^{\mathrm{t}} \theta\right]$,} \\
\hline & 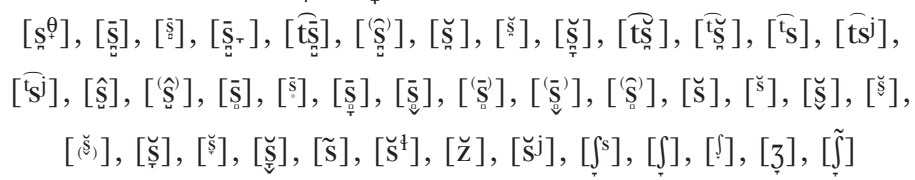 \\
\hline DORSAL & {$\left[\mathrm{x}^{\hbar}\right]$} \\
\hline FARÍNGEO & {$\left[\hbar^{\mathrm{s}}\right],\left[{ }^{\hbar} \mathrm{s}\right],\left[\hbar^{\varsigma}\right],[\hbar],\left[{ }^{\hbar}\right],[\mathrm{S}],\left[{ }^{\complement}\right]$} \\
\hline
\end{tabular}

Como se ve en el Cuadro 6, la inmensa mayoría de los registros se refieren a articulaciones de carácter CORONAL, pues suponen un total de 1262 documentaciones. Un único caso se ha anotado como DORSAL, aunque su carácter velo-faríngeo podría haber autorizado a incluirlo en el grupo más posterior. Por fin, 61 de los casos transcritos en el Atlas quedan adscritos al articulador FARÍNGEO. Las formas coronales están muy repartidas en diferentes sectores articulatorios, desde interdentales hasta postalveolares, como ya se presentó en el Cuadro 1. Las variantes más comunes son la postinterdento-dental fricativa sorda $[\theta]$, la ápico-dental plana fricativa sorda [s] $]$, la predorso-dental convexa fricativa sorda [ $[\mathbf{s}]$, la lámino-alveolar plana fricativa sorda

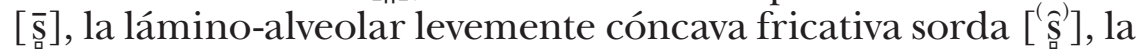

${ }^{31}$ Ya se ha comentado en una nota previa la no participación de [+extendido], dado el carácter faríngeo y no laríngeo de las aspiraciones transcritas; en cuanto a [+constreñido], el único caso de laríngea oclusiva sorda aparece previo a la sibilante, y con la sola mención en el Mapa no es obvia la forma en que ha de interpretarse. Por lo demás, es claro su papel marginal. 
dorso-alveolar convexa fricativa sorda [ $\breve{s}$ ], la dorso-alveolar con-

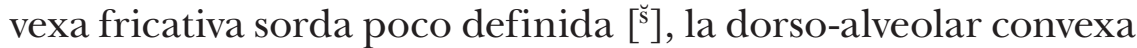
fricativa sonorizada [ $\breve{\mathrm{s}}$ ] y la dorso-alveolar convexa aproximante sorda $[\breve{s}]$.

\section{CuAdro 7}

Rasgos del nodo CORONAL

\begin{tabular}{|c|c|c|}
\hline & {$[+]$} & {$[-]$} \\
\hline [anterior] & $\begin{array}{l}\text { Las demás } \\
\text { coronales. }\end{array}$ & 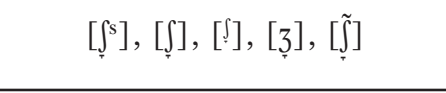 \\
\hline [distribuido] & $\begin{array}{l}\text { Las demás } \\
\text { coronales. }\end{array}$ & 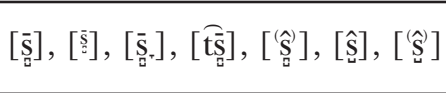 \\
\hline
\end{tabular}

Tanto [anterior] como [distribuido] están circunscritos al nodo CORONAL, así que sólo se especifican para los sonidos dotados de este LUGAR de articulación. De entre todas las coronales hubo sólo 40 registros de postalveolares, que son por tanto [-anteriores]. Menos un par de documentaciones sonoras, la inmensa mayoría de los casos se manifestaron como sordos; uno de los ejemplos estuvo nasalizado. Todos los datos de postalveolares son aproximantes, y de hecho la mayoría son del tipo [S], postalveolar aproximante sorda, que es como se ha adaptado la forma transcrita en el Atlas como [h], dorsopostalveolar sorda muy abierta. En algunas de las consideraciones fónicas y geolingüísticas, como se verá infra, conviene agruparlas con las formas faríngeas, en oposición a las variantes caracterizadas como CORONAL [+anterior].

Se han documentado 118 ejemplos de [-distribuido]. El rasgo [distribuido] permite contrastar sonidos apicales y laminales en diferentes lenguas (por ejemplo, en lenguas de Australia y de la India), tanto en sonidos [-continuos] como en [+continuos], [+nasales] y [+laterales] ${ }^{32}$; en los datos ahora considerados, casi todos los ejemplos [-distribuidos] son de alófonos [+continuos], salvo en un ejemplo documentado de [t $\bar{s}]$. De hecho, la variante más común, con mucho, entre los [-distribuidos], es la ápico-dental plana fricativa sorda [s] $]$. La asignación [+distribuido] etiqueta una constricción lingual extendida por una distancia considerable a lo largo del flujo de aire, mientras que [-distribuido] se refiere a una constricción lingual extendida sólo en una

32 T.A. Hall, art. cit., p. 325. 
distancia breve ${ }^{33}$. La brevedad de la constricción en los sonidos apicales hace que se etiqueten como [-distribuidos], frente a los laminales, dotados de una constricción más larga y en consecuencia [+distribuidos] (id.). Los ejemplos [-distribuidos] del Atlas son, por tanto, aquellos sonidos CORONALES descritos como apicales. Muchos de ellos, por otra parte, son soluciones marcadas ${ }^{34}$.

\section{Proyección CUANTITATIVA}

La adecuada interpretación de la variación de (s) precisa tener en cuenta la forma en que se establecieron las ponderaciones cuantitativas en los mapas sintéticos del Atlas. Debe recordarse que las letras ofrecidas junto a cada variante en cada punto "representan el porcentaje aproximado de aparición de cada alófono en el promedio del habla de cada localidad" ${ }^{55}$. A sabien-

33 Elizabeth SAgey, The representation of features and relations in nonlinear phonology, Massachusetts Institute of Technology, Massachusetts, 1986, p. 278.

34 Pues lo esperable es que las combinaciones no marcadas sean las lámino-dentales y las ápico-alveolares, mientras que la mayor parte de los casos considerados ahora son ápico-dentales. "Many languages contrast dental and alveolar stops. This difference is almost always accompanied by a difference in laminality. All four of the possibilities, apical dental, laminal dental, apical alveolar and laminal alveolar occur, but languages rarely have contrasts in which one sound is apical and the other laminal with the contact being made at the same place on the roof of the mouth. In the languages we have investigated, dental stops are usually laminal rather than apical, with contact on both the teeth and the front part of the alveolar ridge, whereas the alveolar stops are often apical, with contact usually on the center of the alveolar ridge" (P. LAdefoged e I. MAdDieson, op. cit., pp. 20-21). Estos autores recalcan que "in general, if a language has only a dental or an alveolar stop, then that stop will be laminal if it is dental and apical if it is alveolar. But generalizations such as these should be treated with reserve. Most textbooks describe French t, d, n, l as laminal dental and English t, d, n, l as apical alveolar [pero se ha mostrado que 20-30\% de una muestra de hablantes de francés] used alveolar gestures for some of these sounds... and similar percentages of Californian English speakers used dental articulations" (ibid., p. 23).

35 Atlas, p. 16. Se aclara que "aproximados por cuanto que no pretendemos que nuestros porcentajes sean necesariamente exactos y matemáticamente acordes con la realidad de cada habla local; media hora de conversación no es siempre lapso suficiente para reunir el número de testimonios de cada alófono necesario para obtener estadísticas y promedios seguros... Por todo ello no hemos querido presentar en los mapas porcentajes numéricos, que hubieran podido dar al lector una idea -falsa- de exactitud matemática; hemos preferido usar letras equivalentes a porcentajes aproximativos" (id., n. 
das de que se trata de cantidades aproximadas, y en consecuencia contienen una dimensión apreciativa, lo cierto es que la única forma de ponderar la dimensión cuantitativa es considerar las cifras expuestas en la tabla de equivalencias incluida en las primeras páginas de la carta geolingüística:

\section{CuAdro 8}

Equivalencias numéricas de las ponderaciones de los mapas sintéticos del Atlas

\begin{tabular}{|c|c|c|c|}
\hline \multirow{2}{*}{ General } & $\mathrm{G}$ & $100 \%$ prácticamente & 1.000 \\
\cline { 2 - 4 } & $\mathrm{G}-$ & $95 \%$ aproximadamente & 0.950 \\
\hline \multirow{3}{*}{ Frecuente } & $\mathrm{F}+$ & $90 \%$ aprox. & 0.900 \\
\cline { 2 - 4 } & $\mathrm{F}$ & $80 \%$ aprox. & 0.800 \\
\cline { 2 - 4 } & $\mathrm{F}-$ & $70 \%$ aprox. & 0.700 \\
\hline \multirow{5}{*}{ Medio } & $\mathrm{M}+$ & $60 \%$ aprox. & 0.600 \\
\cline { 2 - 4 } & $\mathrm{M}$ & $50 \%$ aprox. & 0.500 \\
\cline { 2 - 4 } & $\mathrm{M}-$ & $40 \%$ aprox. & 0.400 \\
\hline \multirow{5}{*}{ Poco } & $\mathrm{P}+$ & $30 \%$ aprox. & 0.300 \\
\cline { 2 - 4 } & $\mathrm{P}$ & $20 \%$ aprox. & 0.200 \\
\cline { 2 - 4 } & $\mathrm{P}-$ & $10 \%$ aprox. & 0.100 \\
\hline \multirow{5}{*}{ Esporádico } & $\mathrm{E}+$ & $7.5 \%$ aprox. & 0.075 \\
\cline { 2 - 4 } & $\mathrm{E}$ & $5 \%$ aprox. & 0.050 \\
\cline { 2 - 4 } & $\mathrm{E}-$ & $2.5 \%$ aprox. & 0.025 \\
\hline
\end{tabular}

Es decir, lo que se hace aquí para efectuar los cálculos incluidos en esta sección ${ }^{36}$ y poder apreciar cuál o cuáles son los tipos de (s) predominantes en el país es regresar a la cuantificación inicial, aunque convertida ahora a una escala de frecuencias relativas, que es la que aparece en la columna más a la derecha del Cuadro 8.

En esos términos, el total de las frecuencias estimadas en el Mapa 26 es de 193.850 puntos; esta cantidad se obtiene al sumar cada una de las cuantificaciones que acompañan a las 1329

35). Considérese, por otra parte, que (s) no es un sonido infrecuente, por lo que cabe suponer que los transcriptores pudieron apoyarse en una base factual suficiente.

${ }^{36}$ Sólo en esta sección, en realidad, porque la proyección geográfica discutida a continuación en el trabajo se apoya en las estimaciones acotadas por medio de letras. 
observaciones incluidas en el Mapa 26. Este total real está muy cerca del total teórico, que hubiera sido de 193, pues son 193 las localidades encuestadas, considerando un punto por locali$\mathrm{dad}^{37}$. De esta forma es posible, de una manera bastante simple, ponderar el peso de un rasgo fónico o de una variante en el conjunto del país. Por ejemplo, los cinco casos en que se documentan elisiones suman apenas 0.150 puntos, lo que supone sólo una $\mathrm{f}=0.0007$ (es decir, $0.07 \%$ del total de menciones), y es posible establecer que en el total del país son casos muy esporádicos (menos que E-, por emplear la terminología del propio Atlas). Se comenta en seguida la cuantificación general de los rasgos fonético-fonológicos, así como de las principales variantes.

\section{Cuantificación por rasgos}

A la luz de la jerarquía de rasgos adoptada, y de la clasificación de las variantes incluidas en el Mapa 26 discutida supra, la difusión cuantitativa de los rasgos fonético-fonológicos marcados es la que se presenta en el Cuadro 9:

Cuadro 9

Proyección cuantitativa de rasgos fónicos marcados involucrados en la variación de (s)

\begin{tabular}{|c|c|c|c|}
\hline rasgo & puntaje & $f(\%)$ & término \\
\hline [+aproximante] & 20.125 & $0.1038(10.38 \%)$ & $\mathrm{P}-$ \\
\hline$[-$ continuo $]$ & 0.525 & $0.0027 \quad(0.27 \%)$ & $\mathrm{E}-$ \\
\hline$[+$ nasal $]$ & 0.100 & $0.0005 \quad(0.05 \%)$ & $\mathrm{E}-$ \\
\hline [+lateral] & 0.050 & $0.0002 \quad(0.02 \%)$ & $\mathrm{E}-$ \\
\hline [-estridente] & 41.300 & $0.2130(21.30 \%)$ & $\mathrm{P}$ \\
\hline [+sonoro] & 8.625 & $0.0444 \quad(4.44 \%)$ & $\mathrm{E}$ \\
\hline CORONAL & 191.500 & $0.9878(98.78 \%)$ & G \\
\hline DORSAL & 0.025 & $0.0001 \quad(0.01 \%)$ & $\mathrm{E}-$ \\
\hline FARÍNGEO & 2.175 & $0.0112(1.12 \%)$ & E- \\
\hline [-anterior $]$ & 1.975 & $0.0101 \quad(1.01 \%)^{1}$ & $\mathrm{E}-$ \\
\hline [-distribuido] & 10.350 & $0.0533(5.33 \%)^{2}$ & $\mathrm{E}$ \\
\hline
\end{tabular}

${ }^{1}$ Lo que supone $\mathrm{f}=0.0103,1.03 \%$, de entre las coronales.

${ }^{2}$ Igualmente, $\mathrm{f}=0.0540,5.40 \%$, de entre las coronales.

${ }^{37}$ Es, o debería ser, un punto por localidad porque en frecuencia relativa equivale a $100 \%$ de documentaciones de (s) en el lugar. Dado que los transcriptores anotan porcentajes aproximados, o estimaciones con letras, hay algunos casos en que estos no alcanzan a sumar $100 \%$ en una localidad, así como ejemplos en que lo sobrepasan. Con todo, la tendencia general, tanto en cada punto como en el Mapa en su conjunto, es mantener sumas parciales de $100 \%$. 
Como puede apreciarse, la difusión de los rasgos más marcados, alejados por tanto de la fidelidad en cuanto a la identidad de rasgos, es en general escasa. Conviene distinguir, en cualquier caso, entre tres grupos de rasgos, atendiendo a la frecuencia con que se presentan en el Cuadro $9^{38}$.

a) Entre los muy escasos (E-) se encuentran [-continuo], [+nasal], [+lateral], DORSAL, FARÍngeO y [-anterior]. Por supuesto, las rasgos no marcados, a los que corresponderían las cantidades complementarias en cuanto a su difusión, son [+continuo], [-nasal], [-lateral], coronAL y [+anterior]:

[-continuo] alcanza apenas $0.27 \%$ de las documentaciones, frente a una abrumadora mayoría de ejemplos [+continuos]. No es imposible que los casos de articulaciones africadas posean una dimensión léxica, pero en el panorama general quedan reducidos a un carácter esporádico. Aparecen casos [-continuos] en forma de africadas plenas en Pijijiapan (Chiapas), Huatusco (Veracruz), Tlazala (México) y Soto la Marina (Tamaulipas), y en forma de africadas con oclusión breve en San Cristóbal de Las Casas (Chiapas), Comitán (Chiapas), San Juan Evangelista (Veracruz), Córdoba (Veracruz), Orizaba (Veracruz), Amatlán (Veracruz), Tecpan de Galeana (Guerrero), Guarachita (Michoacán), Colima (Colima), Sayula (Jalisco), Ojuelos (Jalisco), Calvillo (Aguascalientes), Guanajuato (Guanajuato), Flores Magón (Chihuahua) y Guamúchil (Sinaloa). Podría decirse, entonces, que el rasgo [-continuo] (y las variantes que lo portan) aparecen con cierta insistencia en tres zonas: el sur del país (en especial en Chiapas), el golfo (sobre todo Veracruz) y el occidente. En todo caso, el modelo de probabilidad logística escalonada que cruza [-continuo] y cinco zonas geográficas no resultó significativo ${ }^{39}$. En cambio, un modelo más idealizado, que opone centro-sur (sureste, centro-este y centro-oeste) frente a norte (noreste y noroeste), sí resultó significativo: el sur documenta $1.9 \%$ de [-continuo], y el norte $0.6 \%$; esto supone que el sur concentra $62.1 \%$ de los casos, mientras que el norte nada más recoge $37.9 \%$. Más importante que esto es la emergencia del rasgo en el modelo escalonado; la probabilidad

38 Recuérdese que los porcentajes del Cuadro 9 se refieren a los puntajes ahí mencionados, que estiman en especial la frecuencia en la suma de documentaciones, tal como se ha explicado. No debe confundirse con el porcentaje simple referido a la proporción de registros frente al total de las 1329 documentaciones; procuro aclararlo en los lugares oportunos.

${ }^{39}$ Los resultados en porcentajes y probabilidad de un nivel fueron los siguientes: sureste, $1.8 \%(p=0.595)$; centro-este, $1.8 \%(p=0.590)$; centrooeste, $2.1 \%(p=0.634)$; noroeste, $0.6 \%(p=0.338)$; noreste, $0.5 \%(p=0.298)$. 
de encontrar una forma [-continua] es el doble en el sur del país $(p=0.611)$ que en el norte $(p=0.323)^{40}$. Este aspecto no carece de cierta relevancia.

Absolutamente infrecuentes son los casos de [+nasal] y [+lateral], así como el lugar de articulación doRSAL, reducidos a un puñado de ejemplos. Los casos de nasalización se presentan en Uruapan (Michoacán), Soto la Marina (Tamaulipas) y Valle de Allende (Chihuahua), lo que subraya también en términos geográficos su carácter esporádico, pues no parece haber de entrada algún tipo de patrón en la dispersión de los casos. Los ejemplos de lateralización se apuntan nada más en Ticul (Yucatán). Desde luego, ambas circunstancias, nasalización y lateralización, son lo bastante interesantes como para promover más investigación local que verifique la extensión o persistencia del rasgo. Más o menos lo mismo puede decirse de la única documentación de articulación DORSAL ${ }^{41}$, sólo registrada en San Felipe (Baja California).

Un poco más notorios son FARÍnGEO y [-anterior], siempre dentro del evidente carácter muy espigado de las documentaciones. Obsérvese que el punto de articulación coronal es absolutamente predominante en el país (suma 191.500 puntos, lo que supone una $\mathrm{f}=0.9878$, es decir, $98.78 \%$ de las documentaciones, lo que permitiría otorgarle un carácter de G. Entre los 61 casos adscritos a la zona FARÍNGEA, hay 9 faríngeo-alveolares, 3 faríngeo post-alveolares y 49 faríngeos; se documentan en los estados de Yucatán (Valladolid, Tizimín ${ }^{42}$, Ticul), Campeche (Campeche, Champotón, Mamantel), Tabasco (Emiliano Zapata, Villahermosa, Huimanguillo), Chiapas (Cintalapa, Chiapa de Corzo, Escuintla), Oaxaca (Sola de Vega, Matías Romero), Veracruz (San Andrés Tuxtla, Tlacotalpan, Veracruz, Misantla, Papantla), Puebla (Tetela, Acatlán), México (Tlazala), Morelos (Jojutla), Guerrero (Ciudad Altamirano), Michoacán (Tiquicheo, Zacapu, Uruapan), Guanajuato (Yuriria), Hidalgo (Apan), San Luis Potosí (San Luis Potosí), Tamaulipas (Ciudad Mante, Ciudad Victoria, Soto la Marina), Nuevo León (Monterrey, Aramberri, Doctor Arroyo), Nayarit (Valle de Banderas ${ }^{43}$, Acaponeta), Durango (San Juan del Río, El Palmito) ${ }^{44}$, Coahuila (Saltillo), Chihuahua (Flores Magón,

40 Razón de verosimilitud $=-97.292$; significación $=0.036$.

41 De hecho, la articulación mencionada en el Mapa es velo-faríngea, no sólo velar, así que bien podría haberse sumado este caso a los propios del lugar FARÍNGEO, pero se ha preferido etiquetarlo como DORSAL precisamente para rescatar su carácter de excepción. Más adelante en el trabajo, de todas formas, esta variante se adhiere a las otras posteriores.

${ }^{42}$ Aparecen dos variantes diferentes.

43 Con dos variantes.

${ }^{44}$ En esta localidad se documentan tres variantes diferentes. 
Ciudad Guerrero), Sinaloa (La Cruz ${ }^{45}$, Culiacán, Guamúchil, Los Mochis), Sonora (Bahía Quino ${ }^{46}$, Hermosillo, Baviácora), Baja California Sur (Mulegé) y Baja California (San Felipe, Tijuana). Las formas FARÍNGEAS aparecen, entonces, por todo el país; llama la atención, sin embargo, la abundancia de documentaciones en el sur y sureste, en el golfo y en el noroeste, lo cual sugiere una cierta estructura en el fenómeno (más adelante se discutirá la posible incorporación del hecho al modelo geolingüístico).

En cuanto al rasgo [-anterior], es propio de cuarenta registros, y aparece en una geografía en parte coincidente con la de FARÍNGEO (lo cual puede sugerir vincular ambos rasgos, infra): Quintana Roo (Chetumal), Campeche (Ciudad del Carmen), Chiapas (Escuintla, Tonalá), Oaxaca (Tapanatepec, Zanatepec), México (Ixtlahuaca), Guerrero (Ometepec), Michoacán (La Huacana, Apatzingán), Colima (Cerro de Ortega), Jalisco (Villa Purificación), Aguascalientes (Calvillo), Querétaro (Jalpan), San Luis Potosí (Tamazunchale, Matehuala, Cerritos, Ciudad Valles), Tamaulipas (Santa Teresa, Nuevo Laredo), Nuevo León (Sabinas Hidalgo, Linares), Zacatecas (Río Grande), Nayarit (Jala, Acaponeta), Durango (Mapimí), Coahuila (Múzquiz), Chihuahua (Valle de Allende ${ }^{47}$, Flores Magón, Ciudad Guerrero), Sinaloa $\left(\right.$ Mazatlán $^{48}$ ), Sonora (Ciudad Obregón, Guaymas, Bahía Quino, Baviácora), Baja California Sur (San José del Cabo) y Baja California (Guadalupe Victoria) ${ }^{49}$.

b) Con carácter simplemente esporádico (E) aparecen [+sonoro] y [-distribuido]:

Hay 159 registros marcados con [+sonoro], aunque pueden referirse a documentaciones propiamente sonoras (en 24 datos), sonorizadas (130) o levemente sonorizadas (5). En conjunto, aparecen por todo el país (en 31 entidades de 32 ), y no parece que respondan a un patrón geográfico particular. El cruce entre el carácter sordo o sonoro de los ejemplos y cinco zonas dialectales no resultó significativo como modelo estadístico en el cálculo escalonado de probabilidad logística. Existe, de todos modos, un ligero aumento de las frecuencias de [+sonoro] cuanto más al norte del país ${ }^{50}$. Los casos

45 También tres variantes.

46 Otras tres formas.

47 Se registran dos variantes.

${ }^{48}$ Con tres variantes.

49 También el modelo logístico de [-anterior] se desarrolla más adelante, en particular por medio de la variante de LUGAR postalveolar.

${ }^{50}$ Los porcentajes y probabilidades binomiales de un nivel fueron los siguientes: sureste, 9.6\% ( $p=0.441)$; centro-este, $11.7 \%(p=0.495)$; 
propiamente sonoros aparecen en las siguientes localidades: Ticul (Yucatán), Campeche y Champotón (Campeche), Tlacotalpan y Veracruz (Veracruz), Tetela (Puebla), Jojutla (Morelos), Uruapan (Michoacán), Apan (Hidalgo), Ciudad Victoria (Tamaulipas), Monterrey y Aramberri (Nuevo León), Jala y Valle de Banderas (Nayarit), San Juan del Río y El Palmito (Durango), Saltillo (Coahuila), Ciudad Juárez y Flores Magón (Chihuahua), Mazatlán, La Cruz, Guamúchil y Los Mochis (Sinaloa) y Bahía Quino (Sonora). Una cuestión importante es que casi todos los casos propiamente sonoros son en cuanto a la zona articulatoria FARÍNGEOS, mientras que todos los sonorizados y levemente sonorizados son CORONALES (dorso-alveolares en su inmensa mayoría). Aunque la actividad laríngea y la zona articulatoria implican en principio hechos fónicos situados en esferas diferentes, es claro que al menos en este caso existe una relación entre ambas, y que la variación detectable entre los grados de sonorización queda sumida en las diferencias más generales relativas al LUGAR.

El rasgo [-distribuido], por su parte, está asociado a 118 registros. No resultó significativo en un modelo escalonado con cinco secciones dialectales, aunque en los estados del centro-oeste se documentó bastante menos que en el resto del país: en $5.8 \%$ de las documentaciones presentes en esa zona (22 de entre 377), frente a las del resto, que guarda siempre una proporción de alrededor de $10 \%$ de los $\operatorname{casos}^{51}$. Aparecieron documentaciones superiores a $10 \%$ en las siguientes localidades, agrupadas en sus estados: en Chetumal y Felipe Carrillo Puerto (Quintana Roo), Valladolid (Yucatán), Campeche, Champotón y Ciudad del Carmen (Campeche), Emiliano Zapata, Frontera y Villahermosa (Tabasco), Tuxtla Gutiérrez, Comitán y Tapachula (Chiapas), Zanatepec y Oaxaca (Oaxaca), Córdoba, Jalapa, Perote, Papantla, Tuxpan, Amatlán y Tempoal (Veracruz), Huauchinango y Teziutlán (Puebla), Toluca y Temascaltepec (México), Jojutla (Morelos), Chilpancingo (Guerrero), Morelia y Zacapu (Michoacán), Tecalitlán (Jalisco), Ciudad Valles (San Luis Potosí), Nuevo Laredo (Tamaulipas), Sabinas Hidalgo (Nuevo León), El Salto y Durango (Durango), Sabinas (Coahuila), Valle de Allende, Delicias y Chihuahua ${ }^{52}$ (Chihuahua), Mazatlán y Los Mochis (Sinaloa), Navojoa ${ }^{53}$, Guaymas, Bahía Quino y Hermosillo (Sonora) y La Paz (Baja California Sur).

centro-oeste, $11.7 \%(\mathrm{p}=0.494)$; noroeste, $12.4 \%(\mathrm{p}=0.511)$; noreste, $14.3 \%$ $(p=0.552)$.

${ }^{51}$ Las proporciones porcentuales, y las probabilidades de un nivel, son: sureste, $10.8 \%(p=0.562)$; centro-este, $10.35 \%(p=0.548)$; centro-oeste, $5.8 \%$ $(p=0.396)$; noroeste, $10.2 \%(p=0.544)$; noreste, $9.0 \%(p=0.511)$.

52 Con dos variantes.

53 También con dos variantes. 
c) Se documentan poco $(\mathrm{P}-, \mathrm{P})$, pero ya de forma claramente apreciable, [+aproximante] y [-estridente]:

El rasgo [+aproximante], propio de 187 registros, resultó también significativo en el modelo geográfico de cinco secciones establecido por medio de la probabilidad escalonada. Es favorecido en general en el norte del país, tanto en el noreste $(16.9 \%$ y $\mathrm{p}=0.560)$ como en el noroeste $(17.8 \%$ y $\mathrm{p}=0.574)$, y no es favorecido ni en el sureste $(10.2 \%$ y $\mathrm{p}=0.416)$ ni en el centro-este $(10.6 \%$ y $\mathrm{p}=0.427)$; el centro-oeste ni favorece ni no favorece $(13.8 \% \text { y p }=0.500)^{54}$. El rasgo alcanza una frecuencia media en Mamantel (Chiapas), San Andrés Tuxtla y Otatitlán (Veracruz) y Jalpa (Zacatecas). Aparece poco (P) en 93 puntos: Tizimín (Yucatán), Emiliano Zapata (Tabasco), Tapachula, Escuintla -en dos variantes-, Pijijiapan y Tonalá (Chiapas), Tapanatepec -también dos veces-, Tehuantepec, Totolapan, Pochutla y Pinotepa Nacional -dos formas(Oaxaca), Tlacotalpan, Otatitlán, Veracruz, Huatusco, Papantla y Tuxpan (Veracruz), Huauchinango y Tepeaca (Puebla), el Distrito Federal, Ixtlahuaca, Toluca y Tenancingo (México), Iguala, Tixtla, Ometepec, Cruz Grande, Tres Palos y Tecpan de Galeana (Guerrero), Morelia, Zamora, Uruapan, Tacámbaro y La Huacana (Michoacán), Cerro de Ortega (Colima), Tecalitlán, Sayula, Tecolotlán, Ocotlán -dos variantes-, Guadalajara, Tepatitlán, Jalostitlán, Lagos de Moreno y Ojuelos (Jalisco), Aguascalientes y Calvillo (Aguascalientes), San Luis de la Paz, León y Pénjamo -también en dos variantes- (Guanajuato), Jalpan (Querétaro), Molango (Hidalgo), Río Verde, San Luis Potosí, Salinas, Charcas, Matehuala, Cerritos y Ciudad Valles (San Luis Potosí), Ciudad Mante, Tula y Soto la Marina -en dos- (Tamaulipas), Monterrey, Aramberri y Ciudad Arroyo (Nuevo León), Valparaíso (Zacatecas), Jala, Tepic -en dos variantes-y Acaponeta (Nayarit), El Salto, Durango, San Juan del Río y Mapimí - dos veces- (Durango), Monclova (Coahuila), Valle de Allende, Delicias y Chihuahua (Chihuahua), La Cruz, Culiacán y Guamúchil (Sinaloa), Álamos, Guaymas, Baviácora y Magdalena de Quino (Sonora), San José del Cabo (Baja California Sur) y Guadalupe Victoria (Baja California).

En cuanto a [-estridente] es propio de 506 registros. Con valor medio, sólo se presenta en cinco localidades: Mamantel (Campeche), San Andrés Tuxtla (Veracruz), Otatitlán (Veracruz), Chilpancingo (Guerrero) y Jalpa (Zacatecas), pero aparece con profusión en pocos casos (tipo P), en 181 ocasiones, y desde luego de manera esporádica (todas las restantes). En principio, se

${ }^{54}$ La razón de verosimilitud del cálculo es -535.030 y la significación 
trata de un rasgo difundido por todo el país (aparece en las 32 entidades federativas), pero sí resultó significativo en el cálculo escalonado con el modelo geográfico de cinco secciones. El rasgo [-estridente] es favorecido en el sureste (40.4\% de los registros de la zona, $\mathrm{p}=0.526)$, en el noreste $(40.7 \%, \mathrm{p}=0.530)$ y especialmente en el noroeste $(46.7 \%$ y $\mathrm{p}=0.589)$; y no es favorecido ni por el centro-este $(31.2 \%$ y $\mathrm{p}=0.427)$ ni el centro-oeste $(33.7 \% \text { y } \mathrm{p}=0.454)^{55}$.

\section{Cuantificación por variantes según LUGAR}

Conviene detenerse ahora a evaluar el papel del LUGAR de articulación, y para ello se ha organizado el material en ocho variantes diferentes, tal como se expone en el Cuadro 10:

Cuadro 10

Variantes según LUGAR de articulación

\begin{tabular}{|c|c|c|c|}
\hline LUGAR (casos) & $<$ variante $>$ & puntaje & $f(\%)$ \\
\hline interdentales (114) & $<\theta>$ & 8.400 & $0.0433 \quad(4.33 \%)$ \\
\hline ápico-dentales (109) & $<\overline{\mathrm{S}}>$ & 9.925 & $0.0511 \quad(5.11 \%)$ \\
\hline predorso-dentales (125) & $<\breve{\mathrm{s}}\rangle$ & 10.175 & $0.0524 \quad(5.24 \%)$ \\
\hline lámino-alveolares (304) & $<\overline{\mathrm{S}}>$ & 34.900 & $0.1800(18.00 \%)$ \\
\hline dorso-alveolares (538) & $<\breve{\mathrm{s}}>$ & 124.850 & $0.6440(64.40 \%)$ \\
\hline otras centrales (19) & $<$ otras $>$ & 0.700 & $0.0036 \quad(0.36 \%)$ \\
\hline postalveolares (53) & $<\int_{T}>$ & 2.550 & $0.0131 \quad(1.31 \%)$ \\
\hline faríngeas (62) & $<\hbar>$ & 2.200 & $0.0113(1.13 \%)$ \\
\hline
\end{tabular}

La primera columna se refiere al área articulatoria, después de llevar a cabo algunas simplificaciones que se explicarán en seguida; la segunda registra entre ángulos la forma más común adoptada por la variante; la tercera y la cuarta se refieren al puntaje con respecto a la suma total de 193.850 puntos discutida supra. La variante llamada interdental, tipo $\langle\theta\rangle$, suma 114 casos, e incluye las formas interdentales propiamente dichas, las postinterdento-dentales y las dento-interdentales. Ya me he referido antes a las variantes lámino-alveolar, dorso-alveolar, ápico-dental y predorso-dental; quedan ahora representadas como

${ }^{55}$ La razón de verosimilitud es -873.272 y la significación 0.001 . 
$<\overline{\mathbf{s}}>,\langle\breve{\mathbf{S}}>,<\overline{\mathbf{s}}>$ y $<\breve{\mathrm{s}}>$. En $<$ otras $>$ centrales han quedado incluidos diecinueve casos de ápico-alveolares y dento-alveolares. En la variante post-alveolar, $\langle\delta\rangle$, se recogen formas propiamente postalveolares, así como postalveolo-alveolares, dento-alveolares palatalizadas y dorso-alveolares palatalizadas. Por fin, entre las faríngeas se consideran las faríngeas, las faríngeo-alveolares, las faríngeo-postalveolares y las velo-faríngeas. Todas juntas suman 1324 registros $^{56}$.

En términos de puntaje, las tendencias son muy claras. Casi dos de cada tres (s) son dorso-alveolares, y ellas solas reúnen 124.850 puntos. Sumadas a las lámino-alveolares, resulta que más de cuatro de cada cinco casos $(82.40 \%)$ son alveolares. A cierta distancia vienen los alófonos dentales, aunque juntos concentran más de uno de cada diez casos $(10.35 \%$ en la escala de puntaje). Todas las otras formas juntas (interdentales, otras centrales, postalveolares y faríngeas) suponen apenas $7.25 \%$ del puntaje total disponible en el Mapa 26.

Se ha considerado entonces, en primer lugar, la posibilidad de trazar un modelo para cinco secciones dialectales y ocho variantes según el LUGAR articulatorio. El Cuadro 11 muestra las frecuencias en los correspondientes cruces:

Cuadro 11

Tabla de contingencia según LUGAR y sección geolingüistica

\begin{tabular}{|c|c|c|c|c|c|c|c|c|}
\hline & \multicolumn{5}{|c|}{ Sección geolingüística } & \multirow[b]{2}{*}{ Total } \\
\hline & & & $\begin{array}{l}\text { centro- } \\
\text { este }\end{array}$ & sureste & $\begin{array}{l}\text { centro- } \\
\text { oeste }\end{array}$ & noroeste & noreste & \\
\hline \multirow[t]{9}{*}{ LUGAR } & interdental & Recuento & 21 & 20 & 32 & 27 & 14 & 114 \\
\hline & & $\%$ de LUGAR & $18.4 \%$ & $17.5 \%$ & $28.1 \%$ & $23.7 \%$ & $12.3 \%$ & $100.0 \%$ \\
\hline & & $\%$ de sección & $7.4 \%$ & $12.0 \%$ & $8.5 \%$ & $8.6 \%$ & $7.4 \%$ & $8.6 \%$ \\
\hline & ápico-dental & Recuento & 27 & 17 & 21 & 28 & 16 & 109 \\
\hline & & $\%$ de LUGAR & $24.8 \%$ & $15.6 \%$ & $19.3 \%$ & $25.7 \%$ & $14.7 \%$ & $100.0 \%$ \\
\hline & & \% de sección & $9.6 \%$ & $10.2 \%$ & $5.6 \%$ & $8.9 \%$ & $8.5 \%$ & $8.2 \%$ \\
\hline & $\begin{array}{l}\text { predorso- } \\
\text { dental }\end{array}$ & Recuento & 31 & 10 & 39 & 25 & 20 & 125 \\
\hline & & $\%$ de LUGAR & $24.8 \%$ & $8.0 \%$ & $31.2 \%$ & $20.0 \%$ & $16.0 \%$ & $100.0 \%$ \\
\hline & & \% de sección & $11.0 \%$ & $6.0 \%$ & $10.3 \%$ & $7.9 \%$ & $10.6 \%$ & $9.4 \%$ \\
\hline
\end{tabular}

56 Recuérdese que hay cinco casos de elisión. 
Cuadro 11

Tabla de contingencia según LUGAR y sección geolingüistica (conclusión)

\begin{tabular}{|c|c|c|c|c|c|c|c|}
\hline & & \multicolumn{5}{|c|}{ Sección geolingüística } & \multirow[b]{2}{*}{ Total } \\
\hline & & \multirow{2}{*}{$\begin{array}{c}\begin{array}{c}\text { centro- } \\
\text { este }\end{array} \\
71\end{array}$} & \multirow{2}{*}{\begin{tabular}{r|} 
sureste \\
40
\end{tabular}} & \multirow{2}{*}{\begin{tabular}{|r|}
$\begin{array}{c}\text { centro- } \\
\text { oeste }\end{array}$ \\
88
\end{tabular}} & \multirow{2}{*}{\begin{tabular}{r|} 
noroeste \\
66
\end{tabular}} & \multirow{2}{*}{\begin{tabular}{|r|} 
noreste \\
39 \\
\end{tabular}} & \\
\hline $\begin{array}{l}\text { lámino- } \\
\text { alveolar }\end{array}$ & Recuento & & & & & & 304 \\
\hline & \% de LUGAR & $23.4 \%$ & $13.2 \%$ & $28.9 \%$ & $21.7 \%$ & $12.8 \%$ & $100.0 \%$ \\
\hline & \% de sección & $25.2 \%$ & $24.1 \%$ & $23.3 \%$ & $21.0 \%$ & $20.6 \%$ & $22.9 \%$ \\
\hline dorso-alveolar & Recuento & 112 & 59 & 167 & 120 & 80 & 538 \\
\hline & $\%$ de LUGAR & $20.8 \%$ & $11.0 \%$ & $31.0 \%$ & $22.3 \%$ & $14.9 \%$ & $100.0 \%$ \\
\hline & \% de sección & $39.7 \%$ & $35.5 \%$ & $44.3 \%$ & $38.1 \%$ & $42.3 \%$ & $40.5 \%$ \\
\hline otra central & Recuento & 4 & 2 & 6 & 6 & 1 & 19 \\
\hline & $\%$ de LUGAR & $21.1 \%$ & $10.5 \%$ & $31.6 \%$ & $31.6 \%$ & $5.3 \%$ & $100.0 \%$ \\
\hline & \% de sección & $1.4 \%$ & $1.2 \%$ & $1.6 \%$ & $1.9 \%$ & $0.5 \%$ & $1.4 \%$ \\
\hline postalveolar & Recuento & 6 & 5 & 15 & 17 & 10 & 53 \\
\hline & $\%$ de LUGAR & $11.3 \%$ & $9.4 \%$ & $28.3 \%$ & $32.1 \%$ & $18.9 \%$ & $100.0 \%$ \\
\hline & \% de sección & $2.1 \%$ & $3.0 \%$ & $4.0 \%$ & $5.4 \%$ & $5.3 \%$ & $4.0 \%$ \\
\hline faríngea & Recuento & 9 & 13 & 8 & 24 & 8 & 62 \\
\hline & \% de LUGAR & $14.5 \%$ & $21.0 \%$ & $12.9 \%$ & $38.7 \%$ & $12.9 \%$ & $100.0 \%$ \\
\hline & \% de sección & $3.2 \%$ & $7.8 \%$ & $2.1 \%$ & $7.6 \%$ & $4.2 \%$ & $4.7 \%$ \\
\hline elisiones & Recuento & 1 & 0 & 1 & 2 & 1 & 5 \\
\hline & $\%$ de LUGAR & $20.0 \%$ & $0.0 \%$ & $20.0 \%$ & $40.0 \%$ & $20.0 \%$ & $100.0 \%$ \\
\hline & \% de sección & $.4 \%$ & $0.0 \%$ & $0.3 \%$ & $0.6 \%$ & $0.5 \%$ & $.4 \%$ \\
\hline Total & Recuento & 282 & 166 & 377 & 315 & 189 & 1329 \\
\hline & $\%$ de LUGAR & $21.2 \%$ & $12.5 \%$ & $28.4 \%$ & $23.7 \%$ & $14.2 \%$ & $100.0 \%$ \\
\hline & \% de sección & $100.0 \%$ & $100.0 \%$ & $100.0 \%$ & $100.0 \%$ & $100.0 \%$ & $100.0 \%$ \\
\hline
\end{tabular}

$\chi^{2}=41.192 ;$ sign. $=0.128$.

Como revela el cálculo de $\chi^{2}$, el conjunto de relaciones expuesto en la tabla de contingencias no es significativo en su globalidad. Pueden vislumbrarse, sin embargo, algunas regularidades en los términos en que está distribuida la frecuencia. Se han sombreado las celdillas en que el porcentaje supera el promedio horizontal (hay más proporción de una variante fónica en una sección determinada que el promedio general de apariciones de esa variante) y las celdillas en que se supera el prome- 
dio vertical (cuando una zona presenta un promedio de cierta variante superior al promedio de registros de cualquier variante). Por ejemplo, se ha sombreado $7.8 \%$ de formas faríngeas en el sureste porque es superior a $4.7 \%$ de faríngeas en todo el país; y se marca $21 \%$ para el LUGAR para el sureste, porque éste es superior a $12.5 \%$ de casos de (s) que concentran las localidades de esta región del país. Como puede apreciarse, ambos hechos son siempre simultáneos. En ese sentido descriptivo, puede decirse, entonces, que las variantes tipo $\langle\theta>$ son más frecuentes en el sureste; que donde son menos típicas las $<\overline{\mathbf{s}}>$ ápico-dentales es en el centro-oeste, frente al resto del país; que las predorsodentales $\langle\breve{\text { s}}\rangle$ se registran en especial en el centro (este y oeste) y en el noreste; que las lámino-alveolares $<\overline{\mathbf{s}}>$ aparecen con mayor decisión en el centro del país (este y oeste) y en el sureste; que las formas dorso-alveolares $<\breve{s}>$ se documentan más en el centro-oeste y en el noreste; que las formas etiquetadas como $<$ otras centrales $>$ aparecen sobre todo en el centro-oeste y en el noroeste; que las $\langle\oint>$ son típicas del norte (noroeste y noreste) -al igual que ocurre con las elisiones-; y que las soluciones faríngeas $\langle\hbar>$ se dan sobre todo en el sureste y en el noroeste.

Resulta tentador establecer tal zonificación dialectal a partir de las generalizaciones extraídas a partir de las frecuencias; finalmente, se trata de los datos expuestos en el Mapa 26 y las tendencias están ahí. Sin embargo, un modelo variacionista tiene que ser un poco más exigente para poder establecer con exactitud cuáles son los elementos que tienen que figurar en un modelo geolingüístico del comportamiento fónico; serán esos elementos, precisamente, los que emerjan en una representación fonético-fonológica de los hechos variables.

Es posible establecer diferentes modelos, progresivamente idealizados. Se llevaron a cabo tres principales:

a) El modelo más general posible, formado por las variantes anteriores -trazadas según el LUGAR articulatorio- y las cinco secciones dialectales esbozadas, hace emerger tan sólo a una de las variantes, las formas faríngeas $\langle\hbar\rangle$. En este caso, la variante es favorecida desde el sureste, con una frecuencia de 0.078 y una $p=0.665$, desde el noroeste $(f=0.076$ y $p=0.659)$ y muy levemente, al borde de la neutralidad, desde el noreste ( $\mathrm{f}=0.042$, $p=0.509)$; no es favorecida en el centro-este $(f=0.032, p=0.436)$ y menos aún en el centro-oeste $(\mathrm{f}=0.021, \mathrm{p}=0.337)^{57}$. Obsérve-

57 Razón de verosimilitud = -242.159 ; significación $=0.004$. 
se que en este modelo se han considerado todos los 1329 registros incluidos en el Mapa, sin importar su rango de frecuencia (que puede ser F, M, P, E) ${ }^{58}$.

b) Puede promoverse, entonces, un segundo modelo que sólo considere aquellos registros que sean más frecuentes en cada localidad -y por tanto de alguna manera más representativos. Se llevaron a cabo dos cálculos diferentes. Por un lado, se consideró aquellas documentaciones marcadas como $\mathrm{F}, \mathrm{M}$ y $\mathrm{P}$ (lo que aporta un total de 632 registros); por otro, sólo los casos de F y M (163 registros). La única variante seleccionada ahora desde el modelo escalonado es la que corresponde a las formas ápico-dentales, $\langle\overline{\mathbf{s}}\rangle$ : la variante es muy favorecida en el sureste $(f=0.146, p=0.708)$, bastante en el noroeste $(f=0.095, p=0.596)$ $y$ algo en el centro-este $(f=0.08, p=0.552)$; no es favorecida ni por el noreste $(\mathrm{f}=0.045, \mathrm{p}=0.402)$ ni por el centro-oeste $(\mathrm{f}=0.034, \mathrm{p}=0.332)^{59}$. Las formas faríngeas no son seleccionadas ahora por un motivo muy simple: casi nunca son abundantes, y sólo en dos casos presentaron una frecuencia superior a E. Es decir, aunque muestran un condicionamiento geográfico, tienden de todos modos a ser esporádicas. La variante ápicodental, en cambio, muestra condicionamiento geográfico, pero sólo cuando se consideran los registros no esporádicos. En cuanto al tercer cálculo, con sólo los registros F y M, no seleccionó ninguna variante en especial como dotada de correlación espacial. Considérese que de las 163 documentaciones que pasaron el corte, 153 corresponden a la variante dorso-alveolar $<\breve{s}>$, que es siempre la más frecuente y la que mayor puntaje alcanza. Aunque hay diferencias porcentuales llamativas, pues si el sureste documenta $85 \%$ de casos, el centro-oeste llega a alcanzar $100 \%^{60}$, la variante no resulta significativa desde el punto de vista probabilístico escalonado.

c) A la vista de estos resultados, se optó por poner a prueba un tercer modelo en el que se mantienen todos los 1329 registros del Mapa 26, pero se maneja una distribución de variantes fónicas mucho más idealizada. Se llevaron a cabo dos cálculos diferentes. En el primero se oponen formas "anteriores" $(<\theta\rangle)$,

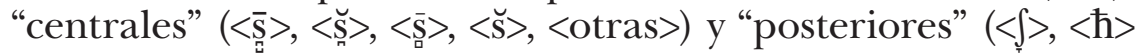
y elisiones). En el segundo, se confrontan "anteriores" $(<\dot{\theta}\rangle,<\overline{\mathbf{s}}\rangle$,

58 No hay casos de G.

59 Razón de verosimilitud $=-161.232$, significación $=0.016$.

${ }^{60}$ Centro-este, $89.5 \%$; noroeste, $92.3 \%$; noreste, $95.5 \%$. 
$<\breve{\mathrm{S}}>,<\overline{\mathrm{S}}>,<\breve{\mathbf{S}}\rangle,<$ otras $>)$ y "posteriores" $\left(\left\langle\int>,<\hbar>\right.\right.$ y elisiones). En la primera versión de este tercer modelo emergieron dos variantes como significativas: las formas "centrales" y las formas "posteriores". Las "centrales" son favorecidas desde el centro del país: en el centro-este $(f=0.869, p=0.580)$ y en el centro-oeste $(\mathrm{f}=0.851, \mathrm{p}=0545)$; y no son favorecidas en el resto de la República (noreste, $\mathrm{f}=0.825, \mathrm{p}=0.497$; noroeste, $\mathrm{f}=0.778, \mathrm{p}=0.423$; sureste, $f=0.771, p=0.413)$. La razón de verosimilitud de este cálculo es -611.697 (sign. $=0.009$ ). En cuanto a las "posteriores", ofrecen casi la imagen de espejo, pues -comparativamenteson preferidas en el noroeste ( $f=0.137, p=0.628)$, en el sureste $(f=0.108, p=0.565)$ y en el noreste $(f=0.101, p=0.545)$, mientras que no son favorecidas ni en el centro-oeste $(\mathrm{f}=0.064, \mathrm{p}=0.422)$ ni en el centro-este $(f=0.057, p=0.392)$-raz. ver. $=-394.953$ (sign. $=0.005)$.

La imagen de espejo puede ser más plena si se da un paso un poco más abarcador, en el camino de establecer un modelo al tiempo más abstracto y más exacto. Si se reúnen en una sola variante todas las formas "no posteriores", estas nuevas "anteriores" también resultan significativas: de nuevo es el centro del país la sección que las prefiere, sea el centro-este ( $\mathrm{f}=0.943$, $\mathrm{p}=0.608)$ o el centro-oeste $(\mathrm{f}=0.936, \mathrm{p}=0.578)$; no las prefieren, en cambio, el noreste $(\mathrm{f}=0.899, \mathrm{p}=0.455)$, el sureste $(\mathrm{f}=0.892, \mathrm{p}=0.435)$ y el noroeste $(\mathrm{f}=0.863, \mathrm{p}=0.372)$. La razón de verosimilitud es ahora -394.953 (sign. $=0.005$ ).

La ponderación de estos dos submodelos puede llevarse a cabo por medio de la razón de verosimilitud, que es más alta cuando se considera a las "anteriores" como la suma de "anteriores" (variante interdental) y "centrales" (raz. ver. $=-349.953$ ), que cuando se considera a las "centrales" solas (raz. ver. = -611.697), y por lo tanto se sugiere un modelo más eficiente ${ }^{61}$.

Una forma de reducir la enorme variabilidad geolingüística en torno al LUGAR articulatorio es proponer que sólo dos tipos de variantes son significativas, las "anteriores" y las "posteriores", de forma que se trataría del único hecho que una proyección dialectal debería atender (al menos con respecto a este rasgo); es también la única realidad que necesitaría una formalización fonético-fonológica.

61 Obsérvese que las cantidades expresadas en los cálculos de la razón de verosimilitud son negativas, lo que hace, obviamente, que -349.953 sea mayor que -611.697 . 
RECAPITULACIÓN: EN BUSCA DEL MEJOR MODELO

Parece necesario detenerse un momento a recapitular los hechos más significativos que han emergido hasta el momento durante el análisis del Mapa 26 del Atlas:

1) La distribución de los hechos es variable, no categórica, y su manifestación es compleja, de modo que no es obvio a primera vista el orden que subyace a los datos. Este orden, sin embargo, existe, y se va revelando según avanza el análisis de los materiales. Es necesario, en consecuencia, un método de carácter variacionista que permita rastrear las estructuras existentes y discernir lo verdaderamente importante de lo secundario. Diferentes hechos pueden describirse en términos de frecuencias y, en ese sentido, ser expuestos por medio de su proyección en ciertas zonas del país. Para establecer la significatividad de las manifestaciones geolingüísticas de los rasgos fónicos, así como de las diferentes variantes alofónicas, es necesario ponderar con precisión las distribuciones. Aquí se ha empleado la asignación de probabilidad logística, que permite establecer modelos cuantitativos sobre los datos.

2) La variante dorso-alveolar $\langle\breve{s}>$ es la más extendida por todo el país, y aunque no es categórica, y hay diferencias entre algunas zonas, no hay indicios de que en alguna gran zona dialectal aparezca significativamente más que en otras. Es la única variante que sobrevive claramente cuando se consideran sólo las documentaciones más frecuentes en las diferentes localidades encuestadas en el Atlas.

3) Las variantes aspiradas, tipo $<\hbar>$, presentan una diferenciación geográfica significativa en un modelo que incluye las 1329 observaciones del Mapa 26. Es decir, en cuanto a su difusión general, sí presentan vinculación geográfica con el modelo de cinco zonas dialectales.

4) Las formas ápico-dentales $<\overline{\mathbf{s}}>$ resultaron significativas en un modelo con las documentaciones de rango F, M y P y las cinco secciones geográficas mencionadas.

5) El rasgo [-continuo] se reveló también significativo, aunque en un modelo muy simplificado en términos de distribución areal, con sólo dos grandes zonas, norte y sur del país.

6) También resultó significativa la distribución de los rasgos [+aproximante] y [-estridente].

7) En cuanto al LUGAR articulatorio, resultó significativa la división de la variable en tres segmentos, ordenado sobre un eje 
antero-posterior: coronales anteriores interdentales; coronales anteriores dentales y alveolares; coronales no anteriores, dorsales, faríngeas y elisiones.

8) También resultó significativa la división de la variable LUGAR en sólo dos secciones: segmentos anteriores (todas las coronales anteriores) vs. segmentos posteriores (todos los demás). Este modelo funciona mejor en los datos que el anterior y al aplicarse sobre una (idealizada) visión en cinco zonas dialectales, divide en dos el territorio mexicano: centro frente a periferia.

Los Cuadros 12 y 13 concentran todas estas observaciones. El Cuadro 12 presenta directamente los atributos probabilísticos alcanzados por cada una de las variantes fónicas (entendidas como rasgos o como alófonos) que se han venido considerando en las sucesivas etapas del análisis, así como la razón de verosimilitud y la significación de cada estrato de cálculos. El Cuadro 13 ofrece una visión más abstracta, establecida en términos binarios, con el propósito de plasmar la zonificación dialectal correspondiente a la variable (s).

Cuadro 12

Secciones dialectales de (s), según probabilidades logísticas escalonadas

\begin{tabular}{|r|c|c|c|c|c|c|c|}
\hline & sureste & centro-este & centro-oeste & noroeste & noreste & raz. ver. & sign. \\
\hline [+aproximante] & 0.416 & 0.427 & 0.500 & 0.574 & 0.560 & -535.030 & 0.047 \\
\hline [-continuo] & \multicolumn{3}{|c|}{0.611} & \multicolumn{2}{|c|}{0.323} & -97.292 & 0.036 \\
\hline [-estridente] & 0.526 & 0.427 & 0.454 & 0.589 & 0.530 & -873.272 & 0.001 \\
\hline FARÍNGEO & 0.665 & 0.436 & 0.337 & 0.659 & 0.509 & -242.159 & 0.004 \\
\hline$<\overline{\mathbf{s}}>$ & 0.708 & 0.552 & 0.332 & 0.596 & 0.402 & -161.232 & 0.016 \\
\hline CORONAL [+anterior] & 0.435 & 0.608 & 0.578 & 0.372 & 0.455 & -394.953 & 0.005 \\
\hline
\end{tabular}

CuAdro 13

Secciones dialectales de (s), en términos binarios

\begin{tabular}{|r|c|c|c|c|c|}
\hline & sureste & centro-este & centro-oeste & noroeste & noreste \\
\hline$[$ +aproximante] & - & - & $-/+$ & + & + \\
\hline$[$-continuo] & + & + & + & - & - \\
\hline$[$-estridente] & + & - & - & + & + \\
\hline FARÍNGEO & + & - & - & + & + \\
\hline$<\overline{\mathbf{S}}>$ & + & + & - & + & - \\
\hline CORONAL [+anterior] & - & + & + & - & - \\
\hline
\end{tabular}


Debe tenerse presente en todo momento que lo ofrecido por los Cuadros no expresa una zonificación categórica, por dos motivos ya mencionados: el carácter muy idealizado de las áreas geográficas planteadas en la construcción del modelo y el carácter probabilístico del que emanan las atribuciones de " + " y “-”. Estamos hablando, por tanto, de tendencias y, sobre todo, de magnitudes comparativas al establecer las distancias lingüísticas entre zonas. Bajo estas consideraciones, las cinco secciones geolingüísticas quedan constituidas para (s) de la manera que se expone a continuación, pues es posible reagruparlas en sólo tres áreas (e incluso en dos):

En una primera gran sección, el norte del país forma una unidad relativamente homogénea. Noroeste y noreste coinciden en todas las atribuciones, menos en una: ambas regiones se caracterizan por la selección de [+aproximante], [-estridente] y FARÍnGEO, y por la no selección de [-continuo] y CORONAL [+anterior]; la única diferencia se encuentra en el empleo de formas ápico-dentales $<\overline{\mathrm{S}}>$, que sí es característico del noroeste, pero no del noreste.

Un segundo bloque está formado por el centro. Las diferencias entre centro-este y centro-oeste son también bastante reducidas. Ambas áreas seleccionan [-continuo] y CORONAL [+anterior], y dejan de lado [-estridente] y FARíngEO. Y aunque el centro-este no favorece las soluciones [+aproximantes], el centro-oeste no las favorece ni las desfavorece. En esos términos, las diferencias claras entre ambas regiones se reducen de nuevo a las preferencias en cuanto al punto de articulación ápicodental, más propio del centro-este que del centro-oeste.

Por fin, el sureste se aparta de las otras dos áreas, pues no selecciona [+aproximante] (en lo que se parece más bien al centro), ni CORONAL [+anterior] (como el norte), y sí selecciona [-continuo] (agrupado con el centro), [-estridente] y FARÍNGEO (como el norte) y las formas ápico-dentales.

Un modelo más idealizado todavía podría incluir sólo dos secciones: centro y periferia, considerando que si se dejan de lado las variantes $<\overline{\mathrm{s}}>$, el centro queda diferenciado del resto del país por seleccionar formas dotadas de CORONAL [+anterior], mientras que la periferia se caracterizaría por [-estridente] y FARÍnGEO. Es posible que tal atribución sea demasiado idealizada, pues deja de lado el comportamiento de [+aproximante] y [-continuo], rasgos para los que el sureste se acerca mucho más al centro. Parece, pues, preferible, decantarse por un reagrupamiento en tres grandes zonas: norte, centro y sureste. 
Punto metodológico central es el hecho de que los datos se pueden agrupar de varias maneras, en busca del modelo geolingüístico óptimo que sea posible trazar alrededor de ellos. En el apartado siguiente se esboza el aspecto general que debería adoptar la fonologización de los datos.

\section{ProyeCGión FONÉTICO-FONOLÓGICA}

En los términos fonológicos de la llamada Teoría de la Optimidad (TO), la distribución de los datos puede exponerse por medio de la contraposición de dos tipos de restricciones, las de fidelidad y las de marcación. Como es bien sabido, se trata de una aproximación más moldeable a los datos variables, en la medida en que permite dar cuenta de la diversidad y en que autoriza la presencia de excepciones -en cierto sentido. Con todo, hay diferencias en la manera en que se manifiestan los datos variables, pues permiten la presencia lícita de varios outputs a partir de un solo input. Se han ofrecido diferentes soluciones al problema, que no se van a discutir aquí, sino que simplemente se presenta una solución operativa ${ }^{62}$.

Las restricciones de fidelidad entre el input y el output regulan la presencia, la identidad de los rasgos y el orden lineal de los segmentos ${ }^{63}$. Las elisiones, por ejemplo, infringen la restricción MAX-IO, pues ésta pide que cada segmento del input tenga un correspondiente en el output. Así, MAX-IO es la restricción que violan los casos de elisión documentados en el Mapa 26 del Atlas $^{64}$. No obstante, dado su nulo papel en los modelos geolingüísticos reseñados, no es necesario incluirla en la descripción fonético-fonológica que sigue, pues sólo se incorporan aquellos elementos que han mostrado tener cierta significación geográfica. La única restricción de fidelidad que es necesario tener en cuenta ahora es la de IDENT [rasgo], que pide que los rasgos del input no sean alterados en el output. Conviene desglosar la restricción genérica en las siguientes restricciones específicas:

62 Véase Martín Butragueño (Variación lingüística y teoría fonológica, El Colegio de México, México, 2002), en especial el cap. 4, para una exposición de algunas de las posibilidades formales para expresar la variación.

63 PAul De LAcy, "Themes in phonology", The Cambridge handbook of phonology, ed. P. de Lacy, Cambridge University Press, Cambridge, 2007, p. 14.

64 También infringen la restricción ONSET, que pide que el ataque de una sílaba esté presente. 
(1) Restricciones de fidelidad:

a. IDENT [aproximante]

b. IDENT [continuo]

c. IDENT [estridente]

d. IDENT [LUGAR]

$e$. IDENT [anterior]

f. IDENT [distribuido]

De esta forma, (1a) sancionará aquellas soluciones que introduzcan una forma dotada con el rasgo [+aproximante], (1b) los casos que resulten ser [-continuo], (1c) los [-estridentes], $(1 d)$ los casos que no sean CORONALEs, $(1 e)$ los [-anteriores] y (lf) los ejemplos dotados de [-distribuido $]^{65}$.

En cuanto a las restricciones de marcación, se ocupan de la buena formación de los outputs. Para el caso es posible postular las siguientes:

(2) Jerarquías para las restricciones de marcación:

a. * ONSET/[+aproximante] $*^{*}$ ONSET/ [-aproximante]

b. * ONSET/[+continuo] $» *$ ONSET $/[$ - continuo $]$

c. *[+estridente] $» *[-$ estridente $]$

d. (*LABIAL, * DORSAL $») *_{\text {CORONAL }}$ * *aríngeO

e. * $[$-anterior $] \gg *[+$ anterior $]$

f. *[-distribuido $] » *[+$ distribuido $]$

Las dos primeras jerarquías son fragmentos de una misma jerarquía de restricciones universalmente fijas, producto de la transformación en restricciones de una de las dos escalas armónicas $(\llbracket \ldots>$ ONSET $/[-$ continuo $]>$ ONSET $/[+$ continuo $]>\ldots \rrbracket)$ surgidas al combinar la escala de prominencia de posición en la sílaba (núcleo $>$ ataque) con la escala de sonoridad (del tipo vocal $>$ líquida $>$ nasal $>$ fricativa $>$ oclusiva) ${ }^{66}$. Esto es, lo que $(2 a)$ dice

${ }^{65}$ Es necesario explicar la introducción del rasgo [-distribuido] en la representación fonético-fonológica de los datos. Aunque [-distribuido] no fue un rasgo seleccionado en el modelo con todos los datos, las formas ápicodentales sí resultaron significativas en un modelo con sólo las documentaciones de carácter F, M y P. La variante ápico-dental, por su articulación apical, es [-distribuida], y suma 109 de los 118 ejemplos que son [-distribuidos]; son, por tanto, la inmensa mayoría de casos. Aunque retomar el rasgo [-distribuido] encierra ahora una simplificación sobre los hechos, permite recuperar sin embargo la mayor parte de lo que hay que decir al respecto.

${ }^{66}$ Véase al respecto JoHn MCCARTHy (A thematic guide to optimality theory, Cambridge University Press, Cambridge, 2002, pp. 17-22) y Francisco 
es que es más grave, o más marcado, un ataque [+aproximante] que un ataque [-aproximante], y lo que (2b) señala es que es más marcado un ataque [+continuo] que un ataque [-continuo].

La jerarquía mencionada en $(2 d)$ se refiere a la marcación del LUGAR y ha sido propuesta por Lombardi ${ }^{67}$. Según $(2 d)$, FARÍNGEO es el lugar de articulación menos marcado, y LABIAL y DORSAL los que más ${ }^{68}$. Hay diferentes argumentos para considerar a CORONAL menos marcado que LABIAL y DORSAL; pueden destacarse su papel en procesos asimilatorios y su carácter central en el espacio articulatorio ${ }^{69}$. Menos evidente es el papel de FARÍNGEO ${ }^{70}$. Una alternativa hubiera sido considerar la restricción HAVE-PLACE, que prohíbe los segmentos sin punto de

Arellanes (El sistema fonológico y las propiedades fonéticas del zapoteco de San Pablo Güilá. Descripción y análisis formal, tesis doctoral, El Colegio de México, México, 2009, pp. 36-39). La escala armónica NuCLEUS/x > NuCleus/y da lugar a la restricción HNUC -nuclear harmony constraint-, que establece que "a higher sonority nucleus is more harmonic than one of lower sonority" (Alan Prince \& Paul Smolensky, Optimality theory. Constraint interaction in generative grammar, Blackwell, Oxford, 2004, p. 20).

${ }^{67}$ Linda Lombardi, "Coronal epenthesis and markedness", Phonology, 19 (2002), p. 221; véase también KAREN Rice, "Markedness in phonology", en The Cambridge handbook of phonology, p. 85.

68 En $(2 d)$ LABIAL y DORSAL aparecen entre paréntesis porque no es necesario considerarlos para los datos de (s), pues no hay ningún caso de LABIAL y uno sólo de DORSAL, que en realidad se puede considerar también FARÍNGEO, pues alude a una variante velo-faríngea.

${ }^{69}$ Para más información, véase Carole Parodis y Jean-François PruNET (eds.), The special status of coronals: Internal and external evidence, Academic Press, San Diego, 1991. Prince y Smolensky (op. cit., pp. 213 ss.) establecen el carácter no marcado de CORONAL.

${ }^{70}$ Hay de hecho formulaciones alternativas; véase por ejemplo KATYA ZubritskAYA ("Mechanism of sound change in Optimality Theory", Language Change and Variation, 9, 1997, p. 124), quien propone *FARÍNGEO" *DORSAL» *LABIAL» *CORONAL. Los argumentos de Lombardi descansan en procesos epentéticos, donde los segmentos que considera FARÍNGEOS son poco marcados. El problema con el planteamiento de Lombardi es que considera FARÍNGEO tanto a los segmentos propiamente FARÍNGEO como a los LARÍNGEOS, de modo que los primeros son [-glotales] y los segundos [+glotales]. De hecho, son los [+glotales] los que son en realidad menos marcados, y se postula una jerarquía del tipo *[-glotal] »*[+glotal]: "The true pharyngeal such as $/ \mathrm{S} /$ are obviously very marked consonants, but my claim is that this must be due to something other than their primary Pharyngeal place" (p. 222). Es claro, por otra parte, a la vista de Cuadros como el 6 (id.) que, para Lombardi, *CORONAL es de todos modos más marcado que [-glotal], de forma que la jerarquía es *CORONAL» *FARíngEO, *[-glotal]» *[+glotal $]$. 
articulación $^{71}$, pero habría que haber supuesto que las transcripciones del Atlas, que hacen referencia a fricativas FARÍnGEAS

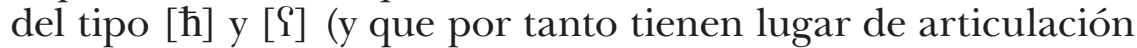
asignado), hubieran de considerarse en realidad aspiraciones laríngeas del tipo $[\mathrm{h}]$ y [h], sin punto de articulación propiamente dicho ${ }^{72}$.

El apoyo para (2c) es de base fonética, dada la complejidad del gesto articulatorio que desencadena las consonantes estridentes, también conocidas como sibilantes o fricativas de obstáculo $^{73}$. En cuanto a (2e) y (2f), el principal argumento para establecer las jerarquías en la dirección que se plantea es intrínseco, en la medida en que la frecuencia de las soluciones menos marcadas es mucho mayor dentro de los materiales ahora considerados ${ }^{74}$. Para (2e) puede defenderse su derivación a partir de una escala natural en la que [+anterior] $>$ [-anterior], de forma que las segundas son más marcadas que las primeras ${ }^{75}$.

${ }^{71}$ La restricción es empleada, por ejemplo, en el análisis de RICHARD E. Morris de la aspiración de /s/ en tres variedades peninsulares ("Constraint interaction in Spanish /s/-aspiration: Three peninsular varieties", en Hispanic Linguistics at the turn of the Millennium: Papers from the $3^{\text {rd }}$ Hispanic Linguistics Symposium, eds. H. Campos, E. Herburger, A. Morales-Front \& T. J. Walsh, Cascadilla, Somerville, 2000, 3.2).

$72 \mathrm{Si}$ se tratara de aspiraciones laríngeas, la jerarquía presentada en $(2 d)$ sería también más convincente -véanse los comentarios de la n. 70.

73 "Fricative sounds may be the result of turbulence generated at the constriction itself, or they may be due to the high velocity jet of air formed at a narrow constriction going on to strike the edge of some obstruction such as the teeth. We will call the latter type sibilants, a term which has been used for a few centuries... More recent terms for these sounds include strident... and obstacle fricatives" (P. LADEFOGED e I. MAdDieson, op. cit., p. 138). En el mismo sentido en que el gesto para las fricativas necesita de una precisión mayor que la requerida para las oclusivas y las nasales (ibid., p. 137), podría pensarse que el gesto para las fricativas estridentes es todavía más específico.

${ }^{74}$ Para (2f) podría explotarse una idea adicional, en la medida en que las formas ápico-dentales están más marcadas -como se mencionó-que las ápico-alveolares (otro tanto ocurriría con las lámino-alveolares, que estarían más marcadas que las lámino-dentales). Pero en este caso la jerarquía adecuada tendría una forma parecida a ésta: *[alv]/[+distr], *[dent $] /[-$ distr $] »$ $*[\mathrm{alv}] /[-\mathrm{distr}], *[\mathrm{dent}] /[+\mathrm{distr}]$, no necesariamente productiva para los datos ahora considerados, teniendo en cuenta que las muy abundantes dorso-alveolares (508 documentaciones) y lámino-alveolares (304) serían también [+distribuidas] y en consecuencia "marcadas".

75 Véase F. Arellanes (op. cit., p. 267), donde se postula la jerarquía *[CORONAL, - anterior] $» *$ [CORONAL, +anterior], independiente del contexto y derivada de una escala natural [CORONAL, +anterior] $>$ [CORONAL, 
Las atribuciones de marcación presentan no pocos problemas ${ }^{76}$ y dado el carácter variable de los datos ahora discutidos -que conlleva, como se ha dicho, la presencia de varias soluciones válidas- quizá hubiera sido interesante acudir a la idea de gramáticas sólo parcialmente ordenadas. Tal problema, sin embargo, no es el núcleo de esta discusión, y bastará por ahora con ponderar las consecuencias de las jerarquías planteadas en el catálogo de (2) para la formalización fonético-fonológica de un modelo geolingüístico de la variación de (s). Los supuestos establecidos hasta el momento se manifiestan entonces en el Cuadro 14:

Cundro 14

Jerarquías de restricciones por secciones geolingüisticas

\begin{tabular}{|c|c|c|}
\hline sureste & centro & norte \\
\hline $\begin{array}{c}\text { IDENT }[\text { aprox }] » * \text { ONSET } / \\
{[\text {-aprox }]}\end{array}$ & $\begin{array}{c}\text { IDENT }[\text { aprox }] » * \text { ONSET / } \\
{[\text {-aprox }]}\end{array}$ & $\begin{array}{c}\text { *ONSET } /[\text {-aprox }] » \text { IDENT } \\
{[\text { aprox }]}\end{array}$ \\
\hline \multicolumn{2}{|c|}{ *ONSET $^{2}[+$ cont $] »$ IDENT [cont] } & IDENT $[$ cont $]$ * *NSET $/[+$ cont $]$ \\
\hline$*[+$ estr] $»$ IDENT [estr] & IDENT [estr] $» *[+$ estr $]$ & $*[+$ estr $] »$ IDENT $[$ estr $]$ \\
\hline *CORONAL » IDENT [LUGAR] & IDENT [LUGAR] $*$ *CORONAL & *CORONAL » IDENT [LUGAR] \\
\hline$*[+$ distr $] »$ IDENT [distr $]$ & - & - \\
\hline$*[+\operatorname{ant}] »$ IDENT $[\mathrm{ant}]$ & IDENT $[$ ant $] » *[\operatorname{tant}]$ & $*[\operatorname{tant}] »$ IDENT $[\mathrm{ant}]$ \\
\hline
\end{tabular}

El Cuadro 14 presenta las repercusiones fonético-fonológicas de las conclusiones geolectales resumidas en los Cuadros 12 y 13, en caso de aceptar el esbozo de restricciones de fidelidad y de marcación propuesto en (1) y (2). Es muy importante dejar claro que lo que ofrece el Cuadro 14 no es la fonología (o la fonético-fonología) de tres secciones geolectales de modo autónomo, sino de forma comparada. Es decir, los resultados habrían sido diferentes de mostrar las restricciones ordenadas de manera que promuevan, en primer lugar, la variante más frecuente; en segundo lugar, la segunda más frecuente, etc., en cada área. Lo que el Cuadro muestra es la fonología compara$d a$, es decir, dónde cierto orden de restricciones adquiere una

- anterior], a propósito del estudio de las fricativas lenis del zapoteco. De hecho, para las necesidades de este trabajo basta con marcar *[-anterior]» *[+anterior], pues el rasgo [anterior] depende de CORONAL.

${ }^{76}$ Cf. K. Rice, art. cit.; Martin Haspelmath, "Against markedness (and what to replace it with)", JL, 42 (2006), 25-70. 
mayor relevancia o, por mejor decir, dónde se invierten los términos de jerarquía entre las dos restricciones contrapuestas. Recuérdese que la relevancia de cada una de las casillas se ha demostrado por medio de su significación probabilística.

El Cuadro 14 debe leerse de la siguiente manera. En lo que toca a la definición del rasgo [aproximante], el centro y el sureste del país dan prioridad en general ${ }^{77}$ a la restricción de IDENTIDAD que pide la preservación del rasgo, frente al norte, donde la restricción de marcación de bajo nivel que pide que el ataque de la sílaba no sea [-aproximante] adquiere mayor relevancia. Para [continuo], el modelo significativo toma en bloque al centro-sureste, frente al norte; a diferencia del rasgo anterior, una restricción de marcación, * ONSET/[+cont], que pide que en el ataque silábico no aparezca un sonido [+continuo], domina en el centro-sureste, frente al comportamiento más fiel del norte. La restricción de marcación *[+estr], que pide que el segmento involucrado no sea estridente, domina en la jerarquía establecida para el sureste y el norte, mientras que el centro es fiel por medio de la restricción de IDENTIDAD de estridencia. Algo parecido ocurre con respecto al LUGAR y la anterioridad; en estos dos casos, el sureste y el norte coinciden en dar prioridad a las restricciones de marcación *CORONAL y *[+ant], que promueven que el segmento no sea coronal y no sea anterior, frente al centro, más fiel y conservador, pues sufraga la restricción de IDENTIDAD. Por fin, sólo se marca una jerarquía específica para el rasgo [distribuido] para el sureste -dando una vez más predominio a la marcación, con *[+distr]-, pues el centro y el norte presentaban comportamientos sesgados (Cuadro 12, supra): el centro-este y el noroeste presentarían la misma jerarquía que el sureste, mientras que el centro-oeste y el noreste se manifestarían como IDENT [distr] $* *[+$ distr].

Resulta interesante observar las diferencias en la constitución del orden de las restricciones en cada una de las zonas de manera global. En el centro, en cuatro de cinco jerarquías predomina la fidelidad; en contraste, sólo en una de cinco en el norte, y sólo en una de seis en el sureste la fidelidad ejerce protagonismo. Si las diferencias más concretas apoyan la presencia de tres zonas, la oposición global entre fidelidad y marcación -si es que tal oposición tiene algo que decir para explicar los proce-

77 En general, pues recuérdese que el centro-oeste ni favorece ni desfavorece el rasgo [+aproximante]. 
sos de variación y cambio lingüístico- refuerza la división entre centro y periferia, conclusión muy general que seguramente no sea difícil de engarzar con hechos históricos (por ejemplo, un poblamiento hispanohablante y sobre todo una urbanización decisiva más tardíos).

\section{Conclusiones}

a) Los hechos relativos a la distribución de (s) son variables y no categóricos. Es decir, si se pretende establecer algunas regularidades dialectales -digamos trazar algún tipo de frontera-, éstas no se constituyen de manera simple y mucho menos de manera categórica. Las variantes están difuminadas por todo el país y prácticamente puede decirse que cualquier alófono puede aparecer en cualquier lugar. Se está hablando, por tanto, de tendencias, que requieren de una metodología específica para establecer las regularidades.

b) Estos hechos variables están, sin embargo, profudamente ordenados y de ninguna manera son caóticos. La enorme variación registrada en el Mapa 26 del Atlas lingüistico de México puede reducirse (regularizarse) enormemente. Es posible hablar de dos o tres zonas geolingüísticas y de un pequeño grupo de rasgos y variantes significativos. Es posible, asimismo, proponer una reducida formalización fonético-fonológica. Frente al exceso etnográfico y la carencia formal-criticados al principio de este trabajo-, es posible desvelar el orden lingüístico y geográfico que subyace en los datos.

c) Las secciones geolingüísticas que han resultado más significativas son tres (noreste, centro y norte) o dos (centro y periferia), según los grados de abstracción con que convenga trabajar. Aunque el aspecto de estas zonas se puede precisar más, y sin duda convendrá hacerlo en el futuro, una insistencia de este trabajo es que la forma apropiada de trabajar con datos variables extremadamente complejos - como es el caso- no es concebir la distribución dialectal como un a posteriori descriptivo, sino como un modelado geolingüístico alrededor del cual se van acumulando argumentos que lo sostengan y pruebas acerca de su significación.

d) Las principales variantes lingüísticas que han resultado significativas son los rasgos [aproximante], [continuo], [estridente], LugAR, [anterior] y [distribuido]. No hay motivo para 
pensar que los elementos útiles para la variación fónica sean distintos a los postulados para dar cuenta de manera universal de cualquier lengua o variedad de lengua. En ese sentido, me parece que es útil buscar la empatía con postulados fonéticofonológicos cuando se trabaja con datos variables. Cuestión diferente es que los hechos de variación y cambio fónico no puedan explicarse simplemente desde la fonología, y que sea necesario apelar a principios sociolingüísticos más generales para explicar los procesos de innovación y difusión.

e) Tampoco es obvio, ni está plenamente solucionado, el modo en que los hechos variables se incorporan a los modelos fonético-fonológicos, ni siquiera en perspectivas recientes más apropiadas para dar cuenta de la diversidad lingüística. En este trabajo, de todas formas, se ha esbozado la forma en que se enlazarían las diferencias geolingüísticas con una aproximación de corte optimal, por medio de la contraposición de dos familias de restricciones, de fidelidad (en especial, de identidad) y de marcación. 


\section{APÉNDICE}

\section{Signos fonéticos utilizados}

\begin{tabular}{|c|c|c|}
\hline Atlas & $A F I$ & Descripción articulatoria \\
\hline$[\theta]$ & {$[\underset{+}{\theta}]$} & interdental fricativa sorda \\
\hline$\left[\theta_{+}\right]$ & {$[\underset{+}{\theta}]$} & interdental aproximante sorda \\
\hline$\left[\underline{\theta}_{+}\right]$ & {$\left[\underset{+}{\theta_{+}}\right]$} & interdental aproximante sonorizada \\
\hline$[\theta]$ & {$[\theta]$} & postinterdento-dental fricativa sorda \\
\hline$\left[\theta_{+}^{+}\right]$ & {$[\theta]$} & postinterdento-dental aproximante sorda \\
\hline$[\hat{\theta}]$ & {$[\widehat{\mathrm{t \theta}}]$} & postinterdento-dental africada sorda \\
\hline$\left[\hat{\theta}^{\prime}\right]$ & {$\left[{ }^{\top} \theta\right]$} & $\begin{array}{l}\text { postinterdento-dental africada sorda con oclusión } \\
\text { breve }\end{array}$ \\
\hline$\left[\begin{array}{c}{ }^{\theta} \\
\mathrm{s}\end{array}\right]$ & {$\left[\mathbf{s}^{\theta}\right]$} & dento-interdental fricativa sorda \\
\hline$[\dot{\dot{\mathrm{S}}}]$ & {$[\overline{\mathrm{s}}]$} & ápico-dental plana fricativa sorda \\
\hline$[\stackrel{\dot{s}}{s}]$ & {$\left[\frac{1}{8}\right]$} & ápico-dental plana fricativa sorda poco definida \\
\hline$\left[\dot{\bar{S}}_{-}\right]$ & {$\left[\overline{\mathrm{s}}_{\mathrm{a}}\right]$} & ápico-dental plana aproximante sorda \\
\hline$[\hat{\overline{\mathrm{s}}}]$ & $\widetilde{[\mathrm{ts}}]$ & ápico-dental plana africada sorda \\
\hline$[\dot{\mathrm{s}}]$ & {$\left[\begin{array}{c}\left(\mathfrak{s}_{\mathrm{a}}\right) \\
]\end{array}\right]$} & ápico-dental levemente cóncava fricativa sorda \\
\hline$[\mathrm{s}]$ & {$\left[\breve{\mathrm{n}^{\mathrm{S}}}\right]$} & predorso-dental convexa fricativa sorda \\
\hline$[\stackrel{s}{s}]$ & {$[\stackrel{5}{\check{s}}]$} & $\begin{array}{l}\text { predorso-dental convexa fricativa sorda poco de- } \\
\text { finida }\end{array}$ \\
\hline 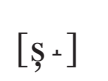 & {$[\breve{s}]$} & predorso-dental convexa aproximante sorda \\
\hline
\end{tabular}




\begin{tabular}{|c|c|c|}
\hline Atlas & $A F I$ & Descripción articulatoria \\
\hline$[\hat{[}]$ & $\widetilde{[\mathrm{tș}}]$ & predorso-dental africada sorda \\
\hline$\left.\hat{\hat{S}^{\prime}}\right]$ & 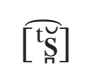 & ntal africada sorda con oclusión breve \\
\hline 1 & {$\left[\mathrm{t}_{\mathrm{s}}\right]$} & dento-alveolar africada sorda con oclusión breve \\
\hline & {$\left[\widehat{\mathrm{ts}^{\mathrm{j}}}\right]$} & dento-alveolar palatalizada africada sorda \\
\hline '] & {$\left[{ }^{{ }^{\top}} \mathbf{s}\right]$} & $\begin{array}{l}\text { dento-alveolar palatalizada africada sorda con } \\
\text { oclusión breve }\end{array}$ \\
\hline & {$[\widehat{\mathrm{s}}]$} & ápico-alveolar cóncava fricativa sorda \\
\hline$\left.\dot{\mathbf{s}}^{)}\right]$ & $\left.[\stackrel{(}{s})^{\prime}\right]$ & ápico-alveolar levemente cóncava fricativa sorda \\
\hline & {$[\overline{\mathrm{s}}]$} & lámino-alveolar plana fricativa sorda \\
\hline & {$[\bar{s}]$} & lámino-alveolar plana fricativa sorda poco definida \\
\hline ] & {$[\overline{\mathrm{s}}]$} & lámino-alveolar plana aproximante sorda \\
\hline & {$[\overline{\mathrm{s}}]$} & lámino-alveolar plana fricativa sonorizada \\
\hline o) 7 & {$\left[\bar{s}^{(-)}\right]$} & lámino-alveolar plano-cóncava fricativa sorda \\
\hline o) 7 & {$\left[\begin{array}{c}(\bar{s}) \\
\stackrel{s}{g}\end{array}\right]$} & lámino-alveolar plano-cóncava fricativa sonorizada \\
\hline & $\left.\left[\mathfrak{s}_{\mathrm{g}}^{(}\right)\right]$ & lámino-alveolar levemente cóncava fricativa sorda \\
\hline [o] & {$[\breve{\mathbf{s}}]$} & dorso-alveolar convexa fricativa sorda \\
\hline & {$[\breve{s}$} & dorso-alveolar convexa fricativa sorda poco definida \\
\hline & {$[\breve{s}]$} & dorso-alveolar convexa fricativa sonorizada \\
\hline & {$[\breve{s}]$} & $\begin{array}{l}\text { dorso-alveolar convexa fricativa sonorizada poco } \\
\text { definida }\end{array}$ \\
\hline
\end{tabular}


Atlas AFI Descripción articulatoria

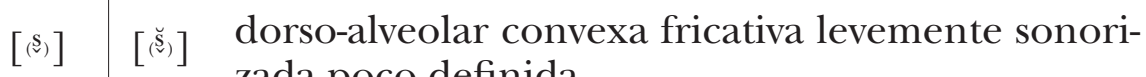
zada poco definida

$\left[\mathrm{s}_{\star}\right] \quad[\breve{s}] \quad$ dorso-alveolar convexa aproximante sorda

[s. $[\stackrel{5}{5}]$ dorso-alveolar convexa aproximante sorda poco definida

$\left[\mathrm{s}_{+}\right] \quad[\breve{s}]$ dorso-alveolar convexa aproximante sonorizada

$[\tilde{\mathbf{s}}] \quad[\tilde{\mathbf{s}}]$ dorso-alveolar fricativa sorda nasalizada

$[\stackrel{8}{\mathrm{~s}}]\left[\breve{\mathrm{s}}^{\ddagger}\right]$ dorso-alveolar convexa lateralizada fricativa sorda

[z] [ $\quad[\breve{z}]$ dorso-alveolar convexa fricativa sonora

$\left[\mathrm{s}^{\prime}\right] \quad\left[\mathrm{s}^{\mathrm{j}}\right]$ dorso-alveolar convexa palatalizada fricativa sorda

$\left[h^{\mathrm{s}}\right] \quad\left[\int^{\mathrm{s}}\right]$ postalveolo-alveolar aproximante sorda con predominio del punto postalveolar

$[\mathrm{h}] \quad\left[\int_{\mathrm{T}}\right]$ postalveolar aproximante sorda

$\left[\begin{array}{lll}{\left[h^{-}\right.} & {[?]} & \text { postalveolar aproximante sorda poco definida }\end{array}\right.$

[G] [3] postalveolar aproximante sonora

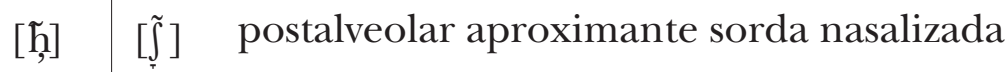

$\left[{ }_{\mathrm{x}}^{\mathrm{h}}\right] \quad\left[\mathrm{x}^{\mathrm{h}}\right]$ velo-faríngea fricativa sorda

$\left[h^{\mathrm{s}}\right] \quad\left[\hbar^{\mathrm{s}}\right]$ faríngeo-alveolar fricativa sorda con predominio del punto faríngeo

$\left[{ }^{\mathrm{h}} \mathrm{s}\right] \quad\left[{ }^{\mathrm{h}} \mathrm{s}\right]$ faríngeo-alveolar fricativa sorda con predominio del punto alveolar

$\left[h^{h}\right] \quad\left[\hbar^{\zeta}\right]$ faríngeo-postalveolar fricativo-aproximante sorda

[h] [ћ] faríngea fricativa sorda 


\begin{tabular}{l|ll}
\hline Atlas & AFI & Descripción articulatoria \\
\hline$\left.{ }^{\mathrm{h}}\right]$ & $\left.{ }^{\mathrm{h}}\right]$ & faríngea fricativa sorda poco definida \\
{$[\mathrm{h}]$} & {$[\mathrm{\complement}]$} & faríngea fricativa sonora \\
$\left.{ }^{[\mathrm{i}}\right]$ & {$\left[^{\mathrm{S}}\right]$} & faríngea fricativa sonora poco definida \\
{$[\varnothing]$} & {$[\varnothing]$} & elisión \\
\hline
\end{tabular}


